\title{
1 Flexible Motor Sequence Generation during Stereotyped Escape Responses
}

2 Yuan Wang ${ }^{1,2, \#}$, Xiaoqian Zhang ${ }^{1,2, \#}$, Qi Xin ${ }^{1,2, \#}$, Wesley Hung ${ }^{3,4}$, Jeremy Florman ${ }^{5}$,

3 Jing Huo ${ }^{1,2}$, Tianqi Xu ${ }^{1,2}, \mathrm{Yu} \mathrm{Xie}^{1}$, Mark J. Alkema ${ }^{5}$, Mei Zhen ${ }^{3,4}$, Quan Wen ${ }^{1,2,6, *}$

$4{ }^{1}$ Hefei National Laboratory for Physical Sciences at the Microscale, Center for Integrative

5 Imaging, School of Life Sciences, University of Science and Technology of China, Hefei, China

$6 \quad{ }^{2}$ Chinese Academy of Sciences Key Laboratory of Brain Function and Disease, Hefei, China

$7 \quad{ }^{3}$ Samuel Lunenfeld Research Institute, Mount Sinai Hospital, Toronto, ON, Canada M5G 1X5;

$8 \quad{ }^{4}$ University of Toronto, Toronto, ON, Canada M5S 1A8

$9{ }^{5}$ Department of Neurobiology, University of Massachusetts Medical School, 364 Plantation 8

10 Street, Worcester, MA 01605, USA

$11{ }^{6}$ Center for Excellence in Brain Science and Intelligence Technology, Chinese Academy of

12 Sciences, Shanghai, China

13 "These authors contribute equally to this work

14 *Corresponding author: qwen@ustc.edu.cn

15 
17 Complex animal behaviors arise from a flexible combination of stereotyped motor primitives.

18 Here we use the escape responses of the nematode Caenorhabditis elegans to study how a 19 nervous system dynamically explores the action space. The initiation of the escape responses 20 is predictable: the animal moves away from a potential threat, a mechanical or thermal

21 stimulus. But the motor sequence and the timing that follow are variable. We report that a 22 feedforward excitation between neurons encoding distinct motor states underlies robust 23 motor sequence generation, while mutual inhibition between these neurons controls the

24 flexibility of timing in a motor sequence. Electrical synapses contribute to feedforward 25 coupling whereas glutamatergic synapses contribute to inhibition. We conclude that $C$. 26 elegans generates robust and flexible motor sequences by combining an excitatory coupling 27 and a winner-take-all operation via mutual inhibition between motor modules.

\section{Keywords}

30 Motor sequence generation, feedforward excitation, mutual inhibition, winner-take-all, escape 31 response 


\section{Introduction}

Nervous systems transform sensation into a sequence of actions. The motor repertoire, constrained by the biomechanics of gait, comprises a finite number of motor primitives that are stereotyped across individuals (Berman, Choi, Bialek, \& Shaevitz, 2014; M. Liu, Sharma, Shaevitz, \& Leifer, 2018; Stephens, Johnson-Kerner, Bialek, \& Ryu, 2008). On the other hand, behavioral flexibility allows an animal to explore the action space, to select better strategies for acquiring reward or avoiding danger in a changing environment (Sutton \& Barto, 2017).

Many factors contribute to behavioral flexibility (Dhawale, Smith, \& Olveczky, 2017; Gordus, Pokala, Levy, Flavell, \& Bargmann, 2015; Remington, Egger, Narain, Wang, \& Jazayeri, 2018). Actions may be generated by an inherently noisy system: synapses are unreliable (Allen \& Stevens, 1994), neurons generate variable spike trains (Mainen \& Sejnowski, 1995), and neural circuits may operate near the edge of chaos (van Vreeswijk \& Sompolinsky, 1996). On the other hand, neural networks, whether adaptive or hard-wired, have structures that shape population neural dynamics onto a low dimensional manifold, where nonrandom and ordered activity patterns emerge (Ganguli et al., 2008; Harvey, Coen, \& Tank, 2012; Inagaki, Inagaki, Romani, \& Svoboda, 2018). Computational models have promised to provide a unified view of these observations (Burak \& Fiete, 2012; Mastrogiuseppe \& Ostojic, 2018; Roberts et al., 2016), but a deep connection between theories and experiments remains to be established.

The initiation of escape responses of the nematode Caenorhabdtis elegans (C. elegans) has long been viewed as an instinctive reflex. Upon a gentle touch to its anterior body, the ventral cord-projecting premotor interneurons AVA/AVD/AVE relay mechanosensory inputs to motor neurons and reliably drive a backward movement (Chalfie et al., 1985; Pirri, McPherson, Donnelly, 
54 Francis, \& Alkema, 2009; Wicks, Roehrig, \& Rankin, 1996). While C. elegans stays committed

55 to its escape decision, the animal remains flexible in its approach to complete the motor sequence.

56 After the reversal, the animal may or may not reorient its body via a deep omega $(\Omega)$ turn, before

57 moving forward (Figure 1A). This allows the animal to resume forward movement in either the

58 original or a new direction. Notably, which action to select and when to execute exhibit trial to

59 trial variability, and they can be coupled. For example, a previous study (Gray, Hill, \& Bargmann,

60 2005) has shown that a longer reversal is likely to be followed by an omega turn.

We sought to understand algorithms and circuit mechanisms for motor sequence generation by investigating recurrently connected interneurons, which are positioned between sensory and motor neurons in the C. elegans nervous system (Figure 2-figure supplement 1A). Previous studies on this layer of neural network (Figure 2-figure supplement 1A) have implicated their roles in exploratory behaviors (Gray et al., 2005; Iino \& Yoshida, 2009; Mori \& Ohshima, 1995; Pierce-Shimomura, Morse, \& Lockery, 1999). During navigation, C. elegans moves towards a new direction by making a reversal and/or a turn in a probabilistic manner. Cell ablation studies revealed that the frequencies of reversals or turns were differentially modulated by many local interneurons including AIB and RIB (Gray et al., 2005). Here we ask whether and how activities of local interneurons and their synaptic interactions shape the dynamics of a motor sequence during escape responses.

Several models have been proposed to account for motor sequence generation. In a class of synaptic chain models (Abeles, 1991; Long, Jin, \& Fee, 2010), feedforward excitation between transiently activated groups of neurons controls the timing of actions hierarchically. Sequential neural activity may also emerge from a cooperation between external inputs and local synaptic interactions in a recurrent network (Rajan, Harvey, \& Tank, 2016; Seeds et al., 2014). We find that 
77 neurons encoding distinct motor states, such as reversal and omega turn, use electrical coupling to 78 reliably drive motor state transitions, whereas they exploit mutual inhibition to flexibly control the 79 timing of an action in a sequence. Our findings provide new insights into how the nervous system 80 organizes time-ordered and variable motor activities, by which stereotyped and flexible animal 81 behaviors emerge. 


\section{Results}

Stereotypical and flexible motor patterns constitute $C$. elegans escape responses

84 A potentially threatening sensory stimulus will trigger an animal's escape response. For example,

85 a gentle touch on the $C$. elegans head, which activates specific mechanosensory neurons

86 ALM/AVM (Chalfie et al., 1985), can induce a reversal or an omega turn (Figure 1A and Video

87 ク).

We quantitatively characterized the escape responses from animals in which channelrhodopsin (ChR2) was expressed in ALM/AVM neurons (Pmec-4::ChR2; lite-1), and optogenetic stimulation was given to the same sensory neurons at a defined light intensity and pulse duration

91 during forward movement (see Materials and Methods) (Leifer, Fang-Yen, Gershow, Alkema, \& 92 Samuel, 2011). ALM/AVM-triggered backward movements responses were robust (only 10\% trials did not respond, Figure $1 C$ left), but subsequent motor sequences constituting each trial varied. Animals exhibited two main types of motor patterns: (1) backward movement was followed by a deep omega turn, and the animal moved forward in a new direction that was different from that before stimulation; (2) an animal executed backward movement and then resumed forward movement in a similar direction as that before stimulation (Figure $\mathbf{1 A}$ and Video ). The head and the tail were diametrically opposed to each other in (1); whereas they were likely motor pattern. 

types of transitions and the corresponding transition rates $r(t)$. Among all reversals survived to time $t, r(t) \Delta t$ computes the fraction of events that will make a transition to another motor state within the time bin $\Delta t$. The type-I (RF) transition rate, $r_{1}$, determines the transition probability from reversal to forward movement; the type-II (RT) transition rate, $r$, determines the transition

108

probability from reversal to omega turn (Figure $1 B$ and Materials and Methods). $r_{1}(t)$ rapidly plateaued in about one second, while $r_{2}(t)$ increased and gradually became the dominant mode (Figure $1 C$ right). The escape responses induced by a focused infrared laser light (Mohammadi, Byrne Rodgers, Kotera, \& Ryu, 2013) exhibited qualitatively similar statistics to ALM/AVMtriggered responses (Figure 1D).

This quantification, which was consistent with a previous observation and description for spontaneous reversals during exploratory behaviors (Gray et al., 2005), confirms the notion that the longer a reversal, the more likely the reversal is followed by a turn.

\section{Local interneurons in the backward module modulate motor state transitions}

We ask how neural dynamics underlie the behavioral variation. Whole brain and multi-neuron calcium imaging of fixed and behaving animals suggested that population interneuron activities, which perform sensorimotor transformation, encode distinct motor states (Gordus et al., 2015;

Kato et al., 2015; Kawano et al., 2011; Li, Liu, Zheng, \& Xu, 2014; Luo et al., 2014; Nguyen et al., 2016; Roberts et al., 2016; Venkatachalam et al., 2016) (Figure $2 A$ and Figure 2-figure 
123

124

125 126

supplement 1B). Several interneurons, including the ventral cord-projecting premotor interneurons AVA and AVE, and the local interneurons AIB and RIM, exhibited increased calcium activity during backward movements (Kato et al., 2015; Laurent et al., 2015; Luo et al., 2014) (Figure 2A and Figure 2-figure supplement 1B).

Structural and functional studies of AIB (Gray et al., 2005; White, Southgate, Thomson, \& Brenner, 1986) indicate that they may play important roles in motor state transitions (Figure 2A). First, AIB establish recurrent connections with the premotor interneurons AVA and AVE that potentiate backward movement either directly through chemical synapses or indirectly through electrical and chemical connections with RIM (Figure 2A). Second, AIB form gap junctions with the inter/motor neurons RIV (White et al., 1986), which play a role in generating a ventral-biased turning behavior (Figure 2A) (Gray et al., 2005). Third, AIB exhibit ramping calcium activity during reversals (Kato et al., 2015; Laurent et al., 2015; Luo et al., 2014), and finally, laser ablation of AIB significantly reduces the frequency of reversals during food search behavior (Gray et al., 2005).

We first examined neuronal correlate of behavioral flexibility in action selection. We compared the AIB ramping activity (Pinx-1::GCaMP6; Pinx-1::wCherry) in different action sequences during either spontaneous or thermal-stimulus-triggered behaviors (Figure $\mathbf{2 B}-\boldsymbol{C}$ and Figure 2-figure supplement $1 \mathrm{~B}-\mathrm{C})$. If the fluorescence signal $\left(\Delta R(t) / R_{0}\right)$ reflects a change of intracellular free calcium concentration $\left[\mathrm{Ca}^{2+}\right]$, the ramping rate, defined as $\zeta=\frac{d R}{d t} \quad$ (Figure $2 C$ ), would be proportional to the calcium current. Higher $\zeta$ may reflect a larger depolarization of the neuronal membrane potential. In Figure $2 \boldsymbol{B}-\boldsymbol{C}, 76 \%$ trials $(91 / 120)$ in the type-I (RF) transition show a positive ramping rate, whereas the proportion rose to $95 \%\left(109 / 115, p<0.0001, \chi^{2}\right.$ test $)$ in 
145 the type-II (RT) transition. Among trials longer than 1.5 seconds, they all showed positive $\zeta$, 146 which during the type-II transition was significantly higher than that during the type-I transition 147 (Figure 2 C and Figure 2-figure supplement $1 C$ ). These results suggest that the more active AIB 148 are, the more likely a worm would terminate its reversal with a turn. reversals followed by omega turns (Figure 2D, Figure 2-figure supplement 1 D and Video 2), whereas strong optogenetic inhibition of AIB (Pmec-4::ChR2; Pnpr-9::Arch; lite-1) during ALM/AVM induced escape responses almost completely abolished omega turns (Figure 2D). We also generated transgenic animals in which AIB are persistently hyperpolarized by an expression of exogenous potassium channels (Pnpr-9::TWK-18(gf)). Interestingly, the no-response fraction increased to $20 \%\left(70 / 332, p<0.0001, \chi^{2}\right.$ test $)$ upon stimulating ALM/AVM in these animals and a significantly larger portion of responses were pauses $\left(35 / 332, p<0.0001, \chi^{2}\right.$ test, Figure $\left.2 \boldsymbol{E}\right)$. We did not observe a significant change in the type-II transition rate $r_{2}$ (Kolmogorov-Smirnov test, $p=0.6$, Figure $2 F$ ), which might be due to a weaker AIB inhibition in these animals. Furthermore, optogenetic ablation of AIB alone (Pnpr-9::PH-miniSOG, Figure 2-figure supplement 1H) (Qi, transition rate (Kolmogorov-Smirnov test, $p<1 \mathrm{e}-20$, Figure 2 -figure supplement 1I). RIM (Figure 2A) also promoted reversal (Figure 2-figure supplement $1 B$ and $\boldsymbol{E}$-G), but were less important in modulating the type-II transition (Figure 2-figure supplement 1D-G). Together, our data strongly suggest that AIB play an important role in promoting reversal and turning behaviors. 

omega turn

How do AIB drive turning behaviors? Whole brain imaging in immobilized animals implicated that AIB and their electrically-coupled partners RIV (Figure $2 A$ and Figure 3-figure supplement 2) exhibited sequentially activated patterns (Kato et al., 2015). We compared RIV activity patterns (Plim-4::GCaMP6) underlying different motor sequences during spontaneous behaviors. During the type-II (RT) transition, RIV calcium signal rose rapidly immediately before 174 a turn began, whereas it remained largely quiescent during the type-I (RF) transition (Figure 3A and Figure 3-figure supplement 1A). The calcium signal decayed towards baseline before the animal finished the turn and resumed forward movement (Figure 3-figure supplement 1B).

To directly probe the functional connectivity between AIB and RIV, we performed 178 simultaneous optogenetic stimulation of AIB (Pnpr-9::Chrimson) and calcium imaging of RIV 179 (Plim-4::GCaMP6::wCherry, Figure 3B). In immobilized wild-type animals, upon stimulating 180 expressed in AIB and RIV (Altun, Chen, Wang, \& Hall, 2009; Bhattacharya, Aghayeva, Berghoff, \& Hobert, 2019). Some of these innexins were shown to form homotypic and/or heterotypic gap junctions (Kawano et al., 2011; P. Liu et al., 2013; Todd A Starich, Xu, Skerrett, Nicholson, \& Shaw, 2009; T. Xu et al., 2018). To determine whether electrical synapses contribute to the observed functional coupling between AIB and RIV, we examined the effect of AIB stimulation in inx-1unc-9unc-7 triple innexin mutants. RIV remained quiescent upon AIB stimulation (Figure $3 C$ red and Figure 3-figure supplement 1D), indicating that gap junction coupling underlies AIB stimulation-mediated RIV calcium activity. 
UNC-7 and UNC-9 are broadly expressed in the motor circuit, and unc-7 or unc-9 mutants exhibit uncoordinated movements that prohibit them from completing a motor sequence (Barnes \& Hekimi, 1997; Brenner, 1974; Kawano et al., 2011; T. A. Starich, Herman, \& Shaw, 1993; T. $\mathrm{Xu}$ et al., 2018). inx-1 single mutants exhibit superficially normal forward and backward movements, allowing us to examine the behavioral requirement of INX-1. The presence of multiple innexins in many $C$. elegans neurons implicates that they may function redundantly at electrical synapses. Consistent with this notion, we find that optogenetic activation of AIB was capable, but with less likelihood, to trigger a turn in inx-1 mutants (Figure $3 D$ ). Rescuing inx-1 in AIB was sufficient to restore the turning probability (Figure 3D). Because inx-1 mutants were still capable of generating omega turns, we propose that either multiple innexins between AIB and RIV, or parallel circuit pathways are at play.

When we performed dual optogenetic activation and calcium imaging in wild-type animals 202 that were allowed to move, RIV calcium activity increase was also observed. But we observed a 203 delay in RIV calcium signal, with its increase arriving at variable times (Figure $\mathbf{3 E}$ and Figure 204 3 - figure supplement $1 F$ ) instead of an immediately rise after stimulation onset. In many trials, the rise of RIV calcium activity coincided with the initiation of an omega turn (Figure 3-figure 206 supplement $1 F$ ), an event that was used to realign all trials at $t=0$ (Figure 3E and Figure 3figure supplement $1 F$ ).

Delayed activation of RIV in a moving animal may result from a convergence of excitatoryand inhibitory- inputs onto the turning module (Figure 2A). When neural activity in behaving animals was aligned to the onset of optogenetic stimulation, a transient quiescence or decrease of

211 RIV calcium activity indeed appeared after $t=0$ (Figure 3F and Figure 3-figure supplement $212 \boldsymbol{I F}$ ). We hypothesized that a rapid increase of calcium activity in Figure $3 \boldsymbol{C}$ (dark blue) could 
213 result from a stronger depolarization of RIV neurons in immobilized animals. Consistently, when

214 the calcium imaging experiment in immobilized animals was combined with a weak and persistent

215 optogenetic inhibition of RIV (Plim-4::Arch), we also observed a delayed and rectified excitation

216 in RIV (Figure 3C light blue and Figure 3-figure supplement 1E).

217 Taken together, our data suggest that the feedforward excitation between the backward 218 module and the turning module takes the form of electrical synapses, likely between AIB and RIV.

219 We considered an effective functional coupling through polysynaptic excitation highly unlikely.

220 First, AIB triggered ventral-biased turning behaviors did not require glutamatergic synaptic

221 transmission (Figure $\mathbf{4 A}$ ), thus excluding polysynaptic pathways via chemical synapses from AIB.

222 Second, two possible polysynaptic excitation pathways from AIB via electrical coupling revealed

223 by the C. elegans connectome (White et al., 1986) involve interneurons RIM or RIS (Figure 3-

224 figure supplement 2). Our optogenetic activation of AIB while inhibiting RIM did not modify the 225 turning probability, whereas activating RIM while inhibiting AIB significantly reduced the turning 226 probability (Figure 2-figure supplement $\mathbf{1 F}-\boldsymbol{G}$ ). Activation of RIS would drive an animal to a 227 pause state and abolish motor actions (Costa et al., 2019). Both results argue against RIM and RIS 228 being directly involved in driving turning behaviors.

231 We next investigated behavioral flexibility in the timing of an action. Given the feedforward 232 coupling between the backward module and the turning module, we asked why omega turns did 233 not immediately follow the optogenetic activation of AIB. We hypothesized that a balance of 234 feedforward excitation and an unknown inhibition provides a potential mechanism to shape the 
235 statistics of the type-II (RT) transition. Besides gap junctions, AIB make chemical synapses with 236 neurons in other modules (Figure $2 A$ and Figure 4-figure supplement 1A), which may facilitate 237 this inhibition. AIB are glutamatergic (Serrano-Saiz et al., 2013). In the glutamate vesicular 238 transport deficient mutant eat-4, optogenetic activation of AIB robustly induced an omega turn 239 that was preceded by a much shorter reversal (Figure 4A left and Figure 4-figure supplement 240 IB). Restoring eat-4 expression specifically in AIB significantly prolonged the reversal length 241 before the onset of an omega turn (Figure 4A left, Figure 4-figure supplement $1 B$ and Video 4). Likewise, stimulating AIB while blocking chemical synaptic transmission from AIB (Pnpr9::TeTx) triggered an omega turn preceded by a shorter reversal (Figure $4 \boldsymbol{A}$ left and Video 4).

244 Consistently, $r 2$ rose more rapidly when glutamatergic inputs from AIB were disrupted (Figure $245 \mathbf{4 A}$ right). channels (Dent, Davis, \& Avery, 1997). Upon optogenetic stimulation AIB, the triple GluCl C. elegans nervous system possesses a family of inhibitory glutamate-gated chloride (GluCl) mutant avr-14(ad1035);avr-15(vu227)glc-1(pk54) exhibited a behavioral phenotype resembling that of the eat-4 mutant (Figure 4B and Figure 4-figure supplement 1B). In some trials (19/112, $p<0.0001$, Fisher's exact test), AIB stimulation immediately triggered omega turns without delay (Video 4). This suggests that postsynaptic $\mathrm{GluCl}$ receptors work synergistically in modulating the onset timing of a turn. Using GFP reporter lines, we found avr-14, glc-1 and avr-15 expressed in many neurons. By focusing on overlaps with neurons known to encode motor states (Figure 2A), 254 we found that Pavr-14 and Pglc-1 reporters exhibited expression in the AIY interneurons, while 255 the Pavr-15 reporter exhibited expression in RIB interneurons (Figure 4C). 
Unlike AIY, RIB receive more and invariant synaptic inputs from AIB (White et al., 1986;

257 Witvliet et al., 2020) (Figure 2A), and hence the prominent and invariant postsynaptic partners of AIB. Consistent with a glutamate mediated feedforward inhibition, RIB calcium activity (Psto$3: \because G C a M P 6)$ significantly reduced upon optogenetic activation of AIB in immobilized animals, which was not observed in the glutamate vesicular transport deficient mutant eat-4 animals (Figure 4D and Figure 4-figure supplement 1C). Moreover, restoring either avr-15 expression in RIB or the avr-14 (or $g l c-1$ ) expression in AIY promoted a longer reversal before the onset of an omega turn (Figure $\mathbf{4 B}$ and Video 4) upon AIB activation.

To investigate the functional connectivity between AIB and RIB, we imaged glutamate 265 signaling (Marvin et al., 2013) at RIB (Psto-3::iGluSnFR) upon persistent optogenetic stimulation of AIB (Pnpr-9::Chrimson; see Materials and Methods). After the onset of stimulation, a rapid rise (Figure $4 \boldsymbol{E}$ inset plot, $\sim 10 \% \triangle F / F_{0}$ ) of iGluSnFR signal on RIB's neurites (Figure $4 \boldsymbol{E}$ and Figure

$$
\frac{\Delta F(t)}{F_{0}}=B\left(e^{-\frac{t}{\tau_{1}}}-e^{-\frac{t}{\tau_{2}}}\right)
$$
where $\tau_{1}=0.9 \mathrm{~s}$, and $\tau_{2}=100 \mathrm{~ms}$. In animals without feeding all-trans retinal (a co-factor required for AIB optogenetic stimulation in this experiment), we observed random and smaller amplitude $\left(\sim 5 \% \triangle F / F_{0}\right)$ fluctuations of iGluSnFR signals (Figure $\left.4 \boldsymbol{E}\right)$. Such dynamics was not observed in the glutamate vesicular transport deficient mutant eat-4 animals (Figure $\mathbf{4 E}$ and

275 Figure 4-figure supplement 2 A bottom). 
277 Local interneurons RIB promote both turning and forward behaviors

278 Local interneurons RIB, together with the ventral cord projection premotor interneurons AVB, 279 have been previously reported to encode forward movement state (Gray et al., 2005; Kato et al., 280 2015; Li et al., 2014). Moreover, RIB form gap junctions with SMDV (Figure 2A), motor neurons 281 that have also been implicated in ventral biased omega turns (Gray et al., 2005; White et al., 1986). 282 RIB calcium activity declined during reversals, and rose during the type-I (RF) and type-II (RT) 283 transitions (Figure 5A and Figure 5-figure supplement 1A), which is characteristic of neurons 284 in both forward and turning modules (Figure $2 \boldsymbol{A}$ ).

Optogenetic manipulation of RIB in freely behaving animals further revealed their functions during motor control. Activating RIB (Psto-3::Chrimson) during reversals triggered a transition to either an omega turn or forward movement (Figure 5B left). Strong optogenetic activation (635 $\mathrm{nm}, 3.75 \mathrm{~mW} / \mathrm{mm}^{2}$ ) of RIB during forward movement reliably triggered omega turns (Figure 5 B right and Video 5). On the other hand, inhibiting RIB (Psto-3::Arch) during forward movement led to a pause (Figure $5 B$ right and Video 5).

When RIB interneurons were directly inhibited to mimic an inhibitory synaptic input, either optogenetically or by an expression of histamine-gated chloride channels (Pokala, Liu, Gordus, \& Bargmann, 2014), the type-II (RT) transition rate $r 2$ plateaued at a significantly reduced value

294 (Figure 5C and Figure 5-figure supplement 1B). Consistently, an escape response comprised 295 of a much longer reversal before an omega turn was initiated (Figure 5C, Figure 5-figure supplement $1 B$ and Video 6), in agreement with the $\mathrm{GluCl}$ rescue results (Figure $\mathbf{4 B}$ left). The 
298 Optogenetic ablation (Psto-3::miniSOG) or blocking chemical synaptic transmission (Psto299 3::TeTx) from RIB also led to prolonged reversals during ALM/AVM-triggered escape responses 300 (Figure 5F). stimulation of AIB (Figure $5 \boldsymbol{D}$ - $\boldsymbol{E})$, the rise of RIV calcium activity in RIB ablated animals showed the same rectified activation when all trials were aligned to the beginning of a turn (Figure $5 \boldsymbol{E}$ ). However, RIV activity was preceded by a longer quiescent state when trials were aligned to the stimulus onset (Figure 5D and Figure 5-figure supplement 1C). Thus, RIB modulate motor state transitions in part through indirect modulation of the timing of RIV activation (Figure 2A).

\section{Inhibitory feedback contributes to reversal termination}

309 The beginning of a turn marks the end of a reversal. We next asked whether the type-II (RT) transition can be accounted for by self-termination of neural activity in the backward module

311 (Figure 2A), analogous to a feedforward synaptic chain model, or, whether activation of the 312 turning module provides a feedback to terminate the activity in the backward module. 
318 patterned illumination during escape responses promoted significantly longer reversals (Figure $319 \boldsymbol{6 B}$ and Video 6).

Observations from optogenetic ablation of RIV/SAA/SMB (Plim-4::miniSOG) also argue against a pure feedforward synaptic chain model. The type-II transition was abolished since animals could no longer generate a complete omega turn (Figure 6C upper panel), while the ability 323 for animals to directly transition from backward to forward movements remained unaffected and 324 the type-I (RF) transition rate $r_{1}$ remained similar (Figure 6 C upper panel) to wild-type animals. 325 Notably, the reversal duration became much longer and approached 30 seconds in some trials, which had not been observed in wild-type animals (Figure 1C, 6C upper panel and Video 3). These results indicate that during normal type-II transitions, persistent neural activity in the upstream backward module could be abolished through inhibitory feedback from the downstream activity in the turning module.

Both the type-I (RF) transition rate (Figure $6 C$ upper panel) and the mirror transition rate (FR) from a forward movement to a spontaneous reversal in wild-type animals (Figure 6 C bottom panel) are consistent with the homogeneous Poisson process at long timescale, leading to exponential survival functions (Figure 6C insets) - fraction of backward or forward movements survived to $t$ (Berg, 1993; Stephens, Bueno de Mesquita, Ryu, \& Bialek, 2011). We did not observe an exponential survival function of reversals in wild-type animals. In the absence of the turning module, the statistics of forward and backward movements (Figure $6 \boldsymbol{C}$ ) became consistent with a 337 simple dynamic model, where a system stochastically transitions between two attractor states with 338 constant rates. 
Together, our data suggest that the feedforward inhibition (Figure 4) and feedback inhibition

340 (Figure 6) between the backward module and the turning module implement a winner-take-all

341 computation for action selection. The motor module with the highest level of activity stays active

342 by suppressing the activities of other modules.

\section{A biophysical model of the type-II transition}

345 With both structural and functional evidence, we now propose a mathematical model for the type-

346 II (RT) transition. The turning module, represented by RIV inter/motor neurons, receives opposing

347 excitatory and inhibitory inputs during backward movements (Figure 7A). The rapid increase of

348 RIV activity coincides with the beginning of an omega turn (Figure $3 \boldsymbol{A}, \mathbf{3 E}$ ). To capture the

349 essential process, we assumed that the membrane potential of RIV $x$ fluctuates around a balanced

350 state $x_{0}$ during a reversal (Figure $7 \boldsymbol{B}$ ), and its neural dynamics is governed by the Langevin

351 equation:

$$
\frac{d x}{d t}=-k\left(x-x_{0}\right)+\eta, \quad x<x_{t h} \quad E q .(2)
$$

353 where $k$ depends on, among others, the gap junction and inhibitory synaptic conductances (see 354 Appendix); and $\eta$ could be regarded as fluctuations in synaptic currents (Lindsay, Thiele, \& 355 Lockery, 2011; Narayan, Laurent, \& Sternberg, 2011) and other sources of noises that are not 356 explicitly considered in the model. For simplicity, $\eta$ is treated as uncorrelated Gaussian white noise: $357\left\langle\eta(t) \eta\left(t^{\prime}\right)\right\rangle=2 \sigma^{2} \delta\left(t-t^{\prime}\right)$. Once the membrane potential crosses the threshold $x_{t h}$, RIV become 358 rapidly depolarized due to a nonlinear rectified activation (Figure $7 \boldsymbol{B}$ ), immediately terminating 
the reversal via feedback inhibition and starting a turn by activating ventral muscles (Figure 7A and 7D).

The next step is to calculate the type-II transition rate $r_{2}$ : the probability that $x$ crosses $x_{t h}$ per unit time. It is currently impossible to measure $k$, but we can proceed by making a non-trivial 363 prediction. Based on electrophysiological recordings of C. elegans interneurons (Lindsay et al., 2011; Roberts et al., 2016), the membrane time constant of a neuron ( 10 milliseconds $)$ is much smaller than the behavioral timescale ( seconds). As a result, the membrane potential of a model neuron rapidly approaches the stationary state $x_{0}$. By solving this problem analytically using onedimensional Fokker-Planck equation near the system equilibrium (see Appendix), we find

$$
r_{2} \approx \frac{k}{\pi} e r f i^{-1}\left[\sqrt{\frac{k}{2 \sigma^{2}}}\left(x_{t h}-x_{0}\right)\right]
$$

Here $\operatorname{erfi}(x)=\frac{2}{\sqrt{\pi}} \int_{0}^{x} e^{z^{2}} d z \quad$ is the imaginary error function.

Eq. (3), however, would lead to a constant rate on the behavioral timescale, like that during 371 the type-I (RF) transition, as expected and confirmed by our computer simulation (Figure 7 C). To explain the experimental observation of the rising phase of $r_{2}$ (Figure $\left.1 C-D\right)$, we incorporated a 373 plasticity mechanism analogous to short-term synaptic depression (STD): the feedforward 374 inhibition from the backward module to the turning module becomes weaker as the reversal lasts longer (Figure 7D). Consequently, the membrane potential moves towards the excitation threshold $376 x_{t h}$ to potentiate transition, allowing the analytical expression for $r_{2}$, Eq. (3), to become time377 dependent. Our hypothesis is consistent with the decay of the glutamate sensor signal on RIB neurites upon AIB stimulation (Figure 4E), an observation that may be explained by a depletion 379 of available vesicles for release at the presynaptic site. Note that calcium activity in AIB cell body, 
380 like that during a reversal (Figure 2 B), kept increasing during persistent optogenetic stimulation 381 (Figure 4-figure supplement $2 \boldsymbol{B}$ ), arguing against the possibility that an opsin-mediated 382 membrane depolarization in the presynaptic neuron undergoes depression upon continuous light 383 activation.

384 By incorporating the exponential decay of inhibitory synaptic strength (Eq. 1), we found that 385 the functional form of the transition rate (see Appendix) can be approximated by

$$
r_{2}(t) \approx k_{1} \operatorname{erfi}^{-1}\left(k_{2}+k_{3} e^{-t / \tau_{g}}\right), \quad E q .(4)
$$

387 where $\tau_{g}=0.9 \mathrm{~s}$ is the decay constant of the glutamate signal (Figure $4 E$ ). The experimentally 388 measured type-II transition rate is well fit by Eq. (4), whereas the standard sigmoid function, $389 \frac{1}{k_{1}+k_{2} e^{-t / \tau}}$, with the same number of free parameters provides a worse fit at the short timescale 390 (Figure 7E). 


\section{Discussion}

393 Complex motor behaviors arise from continual selection and transition among a number of motor 394 primitives. Classic synaptic chain models, in which stereotyped motor sequences arise from 395 feedforward excitation between different groups of neurons, are thought to underlie several motor 396 behaviors such as Zebra Finch singing (Long et al., 2010). A feedforward synaptic chain may 397 underlie the replay of spatiotemporal activity patterns in hippocampus during sleep (Louie \& 398 Wilson, 2001; Skaggs \& McNaughton, 1996), and generate temporally precise firing patterns that 399 correspond to different actions in the motor cortex of behaving monkeys (Shmiel et al., 2006). 400 Alternatively, when several mutually inhibited modules are co-activated by sensory inputs, motor 401 sequences could also emerge by a winner-take-all strategy, a proposed mechanism for the 402 grooming behavior in Drosophila (Seeds et al., 2014). In mice, mutually inhibitory neurons in the 403 central amygdala have been shown to regulate dimorphic defensive behaviors — flight or freezing 404 - triggered by looming visual stimuli (Fadok et al., 2017). Here, we find the two schemes are 405 likely integrated by the C. elegans nervous system to generate robust and flexible motor sequences 406 (Figure 7D).

In C. elegans, feedforward excitation between the backward module and the turning module

408 (Figure 7A) can reliably trigger an omega turn followed by forward movement through strong and 409 persistent activation of local interneurons AIB (Figure 2D and Figure 2-figure supplement 1D).

410 In other words, the action in a motor sequence can be selected through feedforward excitation, 411 triggered by either external sensory stimulus or fluctuations of internal circuit dynamics. The 412 timing of an action can be tuned by augmenting the feedforward excitation with glutamatergic 413 feedforward inhibition between AIB and RIB (Figure 7A), likely through modulating the strength 
414 of inhibitory inputs through short term synaptic plasticity. Previously, a tyraminergic feedforward 415 inhibition (Alkema, Hunter-Ensor, Ringstad, \& Horvitz, 2005; Pirri et al., 2009) from the RIM 416 interneurons in the backward module to the SMD motor neurons in the turning module was shown 417 to suppress head movement during reversals. We propose that these functional motifs 418 feedforward excitation and inhibition — are combined with a nonlinear activation of turning 419 neurons (Figure $7 D$ ) to produce flexible type-II (RT) transitions.

A simple synaptic chain model predicts that abolishing neural activity in a downstream 421 module would not directly affect upstream neural output. However, when RIV/SAA/SMB in the 422 turning module were ablated or inhibited (Figure $6 \boldsymbol{B}-\boldsymbol{C})$, we observed prolonged reversals during 423 escape responses. Hence, the turning module may provide feedback inhibition onto the backward 424 module, contributing to the reversal termination during type-II transitions. The cellular and 425 molecular mechanisms for inhibitory feedback remain to be identified. One possible 426 implementation is cholinergic synaptic outputs from SAAD onto RIM and AVA interneurons in 427 the backward module; another possibility is synaptic outputs from RIB onto AVA/AVE (Figure 428 7A). Together, the feedforward coupling between the backward module and the turning module 429 facilitates a defined sequential activity pattern, whereas the winner-take-all operation through 430 mutual inhibition between the two modules avoids an action conflict.

Sensorimotor transformation depends on the initial condition of the network state (Remington, 432 Egger, et al., 2018; Remington, Narain, Hosseini, \& Jazayeri, 2018). We show that when the 433 backward motor state is suppressed via the hyperpolarization of interneurons AIB, an identical 434 mechanosensory stimulus is less likely to elicit an escape response (Figure $2 \boldsymbol{E}$ ). A recent study 435 also demonstrated that mechanosensory stimuli were unlikely to drive other motor programs when 
C. elegans was executing a turn (M. Liu et al., 2018). We propose that the inhibition from the turning module to the backward module (Figure $7 D$ ) may account for this observation.

We view omega turn, a motor state encoded by transient activity in RIV, as a special manifold connecting two attractors represented by persistent activity in the forward or backward module

(Figure 7D). In our simplified model, neurons within a module were treated as a homogeneous population. Nevertheless, interneurons with heterogeneous functional properties have been found. For example, laser ablation of AIB and RIM in the backward module (Figure 2A) differentially affect the probability of spontaneous reversals (Gray et al., 2005). While RIM showed increased

444 calcium activity during reversals (Figure 2-figure supplement 1B) and promoted reversal 445 duration upon optogenetic activation (Figure 2-figure supplement $1 \boldsymbol{E}$-G), they were less important in modulating the type-II transition than AIB did (Figure 2-figure supplement 1D-G). The impact of functional heterogeneity on the attractor dynamics and motor state transitions remains to be understood. variability. We speculate that stochasticity in neural dynamics and behaviors may allow animals to efficiently explore the action space (Dhawale et al., 2017; Tumer \& Brainard, 2007); learning, by which functional connectivity between motor modules is modified through synaptic plasticity, may optimize action selection and timing (Sutton \& Barto, 2017). We found that in C. elegans, the

454 functional connectivity between motor modules comprises of feedforward excitation and mutual 455 inhibition. Conserved network motifs may be distributed among mammalian forebrain and 456 midbrain circuits (Fadok et al., 2017; Klaus, Alves da Silva, \& Costa, 2019). Other animals could 457 use similar algorithms to organize neuronal activities into sequential states to drive motor 458 primitives, by which stereotyped and flexible behaviors emerge. 


\section{Acknowledgments}

460 We thank Louis Tao for discussions, Pinjie Li for analyzing the reorientation angles during the 461 type-I and type-II transitions. This work was funded by National Science Foundation of China 462 Grants NSFC-31471051 and NSFC-91632102, the Strategic Priority Research Program of the 463 Chinese Academy of Sciences (Pilot study, grant XDPB10, Grant No. XDB39000000), the 464 Guangdong Province with Grant No. 2018B030338001, the CAS Hundreds Talent Plan, and the 465 Fundamental Research Funds for the Central Universities (QW), and the Canadian Institute of 466 Health Research Foundation Scheme 154274 (MZ). Mei Zhen thanks her lab members Yangning 467 Lu, Maria Lim, and Jyothsna Chitturi for sharing their unpublished optogenetic methods, 468 observations, and reagents used in this study.

470 Declaration of Interests

471 The authors declare no competing interests. 


\section{Materials and Methods}

\section{Key resources table}

\section{Key Resources Table}

\begin{tabular}{|c|c|c|c|c|}
\hline $\begin{array}{l}\text { Reagent type } \\
\text { (species) or } \\
\text { resource }\end{array}$ & Designation & $\begin{array}{l}\text { Source or } \\
\text { reference }\end{array}$ & Identifiers & $\begin{array}{l}\text { Additional } \\
\text { information }\end{array}$ \\
\hline $\begin{array}{l}\text { Strain, strain } \\
\text { background ( } C \text {. } \\
\text { elegans) }\end{array}$ & $\begin{array}{l}\text { C. elegans } \\
\text { strains used } \\
\text { and generated } \\
\text { in this study }\end{array}$ & $\begin{array}{l}\text { Caenorhabditi } \\
\text { s Genetics } \\
\text { Center (CGC) } \\
\text { and this } \\
\text { paper }\end{array}$ & & Supplementary File 1 \\
\hline $\begin{array}{l}\text { Recombinant } \\
\text { DNA reagent }\end{array}$ & $\begin{array}{l}\text { Plasmids } \\
\text { generated in } \\
\text { this study }\end{array}$ & This paper & & Supplementary File 2 \\
\hline $\begin{array}{l}\text { Recombinant } \\
\text { DNA reagent }\end{array}$ & $\begin{array}{l}\text { Primers for } \\
\text { genes (or } \\
\text { promoters) } \\
\text { used in this } \\
\text { study }\end{array}$ & This paper & & Supplementary File 3 \\
\hline $\begin{array}{l}\text { Software, } \\
\text { algorithm }\end{array}$ & MATLAB & Mathworks & RRID:SCR_001622 & $\begin{array}{l}\text { https://www.mathworks. } \\
\underline{\text { com }}\end{array}$ \\
\hline $\begin{array}{l}\text { Software, } \\
\text { algorithm }\end{array}$ & $\begin{array}{l}\text { CoLBeRT } \\
\text { system }\end{array}$ & Samuel Lab & & $\begin{array}{l}\text { http://colbert.physics.har } \\
\underline{\text { vard.edu/ }}\end{array}$ \\
\hline $\begin{array}{l}\text { Software, } \\
\text { algorithm }\end{array}$ & ImageJ & $\begin{array}{l}\text { Media } \\
\text { Cybernetics }\end{array}$ & RRID:SCR_003070 & https://imagej.net/ \\
\hline $\begin{array}{l}\text { Software, } \\
\text { algorithm }\end{array}$ & LabVIEW & $\begin{array}{l}\text { National } \\
\text { Instruments }\end{array}$ & RRID:SCR_014325 & http://www.ni.com \\
\hline
\end{tabular}

474

C. elegans strains including wild-type (N2), mutants, and transgenic worms were grown and

477 cultivated according to standard procedures (Brenner, 1974). All strains used in this paper can be 
478 found in the Supplementary File 1. Transgenic worms for optogenetic experiments were cultivated

479 in dark on NGM plates with OP50 bacteria and $0.4 \mathrm{mM}$ all-trans retinal (ATR) for over 5 hours.

480 We used young adult hermaphrodites to perform optogenetic and calcium imaging experiments,

481 and L4 hermaphrodites to obtain expression patterns.

\section{Molecular biology}

483 Standard molecular biology methods were used. Details of plasmids, the promoters and rescue 484 genomic DNA (or cDNA) sequences can be found in Supplementary File 2-3.

485 Optogenetics

486 Worms were first washed in M9 buffer (or transferred onto an unseeded NGM plate for 1-3 487 minutes), then transferred onto a fresh agar plate [ 0.8\% (w/v) agar in M9 buffer, without food], 488 mounted on a motorized stage. Worms were left to freely explore the new environment for 3-5 489 minutes before testing, and were automatically tracked and retained within the field of view of a 490 10× objective (Nikon Plan Apo, NA = 0.45) mounted on an inverted microscope (Nikon Ti-U, 491 Japan) via dark field infrared illumination. Worm behaviors were recorded by a CMOS camera 492 (Basler, aca2000-340kmNIR, Germany). MATLAB custom software (MathWorks, Inc. Natick, 493 MA, USA) was used for post-processing behavioral data and extracting moving directions and the 494 kinematics of omega turns.

495 For freely roaming worms without optogenetic stimulation, we only recorded them for 5-8 496 minutes.

For worms with optogenetic stimulation, lasers and a digital micromirror device (DLI4130

498 0.7 XGA, Digital Light Innovations, TX, USA) were used to generate a defined spatiotemporal 
499 illumination pattern (Leifer et al., 2011) at a specific wavelength (473 nm, $561 \mathrm{~nm}$ or $635 \mathrm{~nm}$ ), and

500 to manipulate the activities of neurons expressing light-activated channels (ChR2, Arch or

501 Chrimson) (Husson et al., 2012; Nagel et al., 2005). To eliminate the effect of adaptation, single

502 worm was stimulated 5-8 times with at least a 50 -second inter-stimulus interval. For example:

503 (1) To trigger escape responses, worms received $1.5 \mathrm{~s}$ blue light (short enough and over $80 \%$ trials

504 showed responses in pilot experiments) to activate mechanosensory neurons ALM/AVM. All

505 worms in the dataset had at least $20 \%$ probability to perform either type-I or type-II transitions. In

506 other cases, $7 \mathrm{~s}$ (or $12 \mathrm{~s}$ ) optogenetic illumination were used to ensure persistent

507 activation/inhibition of local interneurons.

508 (2) In some experiments, worms received sequential optogenetic stimulations with different colors 509 and varying durations controlled by diaphragm shutters (GCI 7102M, Daheng Optics, China). For

510 example, the $1.5 \mathrm{~s}$ blue light optogenetic stimulation (to trigger escape response) was followed by 511 green light with a duration of 3-12 s to inhibit other interneurons in the motor control circuit.

512 (3) We also performed selective optogenetic manipulation of interneurons when their cell body 513 positions were sufficiently apart, given that the lateral resolution of our CoLBeRT system is up 514 to $\sim 5 \mu \mathrm{m}$. For example, RIV and SAAD neurons are separated by at least $20 \mu \mathrm{m}$ along the 515 dorsal-ventral axis (Figure 6A). In order to inhibit SAAD or RIV independently (Plim$5164:$ Arch::GFP), we generated a spatial pattern to selectively illuminate the dorsal or ventral side 517 (Figure $6 B$ right). At the same time, we monitored GFP emission signals from these neurons 518 excited by the laser $(473 \mathrm{~nm})$ to ensure we targeted the correct region (Figure $6 \boldsymbol{B}$ ).

\section{Calcium imaging}


521 protein. Calcium activity was measured as a ratiometric change. For example, neural activity of 522 AIB was measured as a fluorescence ratio of GFP to RFP $\left(\Delta R(t) / R_{0}\right)$ (GCaMP6/wCherry), where $523 R_{0}$ is the baseline ratio. In some cases, we define a new normalized ratiometric measure, $\Delta$ ? $(t)=$ $524 \quad[\Delta R(t)-\Delta R(t=0)] / R_{0}$.

When cell-specific promoters are available, including AIB and RIB, we performed calcium 526 imaging using wide-field fluorescence microscopy. Unrestrained behaving worms were placed on

527 fresh agarose plates [2\% (w/v) agarose in M9 buffer], tracked by a motorized stage using the 528 CoLBeRT system with infrared light illumination (Leifer et al., 2011). Blue and green lights were 529 employed to excite GCaMP6 and wCherry (or mCardinal) proteins. Green and red emission 530 signals were captured by a $10 \times$ objective (Nikon Plan Apo, WD = 4 mm; NA = 0.45, Japan) at 50 531 fps with an exposure time of $20 \mathrm{~ms}$, separated by a dichroic mirror, relayed by an optical splitter

532 (OptoSplit II, Cairn-Research, UK), and projected onto one-half of a sCMOS sensor (Andor Zyla 533 4.2, UK) simultaneously. Green and red channels were aligned and processed by custom-written 534 MATLAB scripts (T. Xu et al., 2018). Single worm was recorded for 3-10 min.

To image RIV neurons, which lack cell-specific promoters, we picked transgenic worms 536 (Plim-4::GCaMP6::wCherry) with stronger fluorescence expression on RIV, and increased the 537 exposure time (up to $50 \mathrm{~ms}$ ) to obtain high signal-to-noise ratio images.

\section{Multi-neuron calcium imaging in a freely behaving worm}

539 To image calcium activity of multiple neurons in a freely behaving worm (e.g., Figure 2-figure 540 supplement $1 B$ ), we combined a spinning disk confocal inverted microscope (Nikon Ti-U \& 541 Yokogawa CSU-W1, Japan) for calcium imaging with a customized upright light path for worm 
542 tracking and behavior recording. A worm was placed on an agarose pad $[\sim 2 \%(\mathrm{w} / \mathrm{v})]$ mounted on

543 a motorized stage. We used a 40× air objective (Nikon Plan Apo, WD 0.25-0.17 mm; NA = 0.95,

544 Japan) or a 60× water immersion objective (Nikon Plan Apo, WD 0.22 mm; NA = 1.20, Japan).

545 The wavelengths of confocal excitation lasers were $488 \mathrm{~nm}$ and $561 \mathrm{~nm}$; the emission lights were

546 split (Andor Optosplit II, UK) in front of a sCMOS camera (Andor Zyla 4.2, UK). At the same

547 time, we utilized a customized light path that was aligned to the same $z$ axis to track the worm and

548 to record behavioral data. In the upright path, a low magnification $10 \times$ objective (Nikon Plan Fluor,

549 WD $16 \mathrm{~mm}$; NA = 0.30, Japan) was used to gather fluorescent light excited by confocal lasers, 550 and the fluorescent signal was processed to identify worm positions. A real time feedback signal

551 was sent to a motorized stage to keep the worm head within the center of field of view. Meanwhile,

552 we illuminated the worm by an infrared ring surrounding the high magnification objective to record 553 worm behavior through the upright light path.

554 Simultaneous optogenetic manipulation and calcium imaging

555 To combine calcium imaging and optogenetic manipulation (e.g., Figure 3B), a 635 nm laser was

556 added to activate Chrimson. Because blue light can also activate Chrimson, excitation light for 557 GCaMP6 imaging and red light for optogenetic stimulation were synchronized using a TTL signal 558 (LabJack Corp., U3-HV) controlled by LabVIEW (National Instruments Corp., USA). For 559 example, to verify the connectivity between AIB and RIV, we activated AIB using red laser (635 $560 \mathrm{~nm}$ ) and recorded the calcium signal in RIV using blue LED excitation (M470L3-C1; Thorlabs, 561 USA) for 7 seconds simultaneously. Both restrained and freely moving worms were tested. 562 Restrained worms were placed on $10 \%$ (w/v) agarose plates with coverslips, whereas freely 563 moving ones were placed on $2 \%(w / v)$ fresh agarose plates. Single worm was stimulated 5-8 times 564 with at least a $50 \mathrm{~s}$ inter-trial interval. 


\section{Thermally-induced escape responses}

566 In addition to optogenetic stimulation of mechanosensory neurons, we also used a thermal stimulus

567 to trigger escape responses. We illuminated the head of a worm with a focused infrared laser light

$568\left(1480 \mathrm{~nm}\right.$; spot diameter $\left.\sim 120 \mu \mathrm{m}, \Delta \mathrm{T} \sim 2{ }^{\circ} \mathrm{C}\right)$ for $0.75 \mathrm{~s}$ (short enough and over $80 \%$ trials showed

569 responses in pilot experiments), and animals responded with reversals or omega turns to avoid the

570 stimulus (Mohammadi et al., 2013). The transition rates were qualitatively similar to ALM/AVM-

571 induced escape responses (Figure $1 \boldsymbol{C}$-D). Identical experimental protocols were used when

572 thermally-induced escape responses were followed by optogenetic manipulation or calcium

573 imaging of interneurons.

\section{$574 \quad$ Glutamate imaging}

575 Glutamate imaging was conducted on worms expressing iGluSnFR (Marvin et al., 2013) on RIB 576 and Chrimson on AIB (Figure $4 \boldsymbol{E}$ and Figure 4-figure supplement 2A). All worms were

577 restrained on fresh $10 \%$ (w/v) agarose plates with coverslips. We used $60 \times$ water immersion 578 objective. Both processes and cell body of RIB were imaged on the same focal plane. Imaging 579 acquisition rate is $150 \mathrm{fps}$. Like calcium imaging, blue excitation light (weak enough to reduce the 580 bleaching effect; M470L3-C1; Thorlabs) for glutamate imaging and red light (635 nm, 6.11 $581 \mathrm{~mW} / \mathrm{mm}^{2}$ ) for optogenetic stimulation were synchronized with a TTL signal. Glutamate signaling 582 resulted in a change of the green fluorescence signal on the membrane of RIB interneurons. 583 Stimulation and imaging sustained for more than 8 seconds. Single worm was stimulated 2-3 times 584 with at least a $50 \mathrm{~s}$ inter-stimulus interval.

\section{Optogenetic ablation}


586 We used miniSOG (mini Singlet Oxygen Generator) to ablate specific neurons in C. elegans. Upon

587 blue light stimulation, a mitochondria-targeted miniSOG (TOMM20-miniSOG) (Qi et al., 2012)

588 and a membrane-targeted miniSOG (PH-miniSOG) (S. Xu \& Chisholm, 2016) were employed to

589 induce cell death in cell-autonomous manner. L2/early L3 worms were transferred onto an

590 unseeded NGM plate, restricted by a small ring of filter paper soaked with $100 \mu \mathrm{M} \mathrm{CuCl}_{2}$. Worms

591 were illuminated for 40-60 min (TOMM20-miniSOG) or 2 min (PH-miniSOG) with blue LED

592 (M470L3-C5; Thorlabs) at the intensity of $0.46 \mathrm{~mW} / \mathrm{mm}^{2}$. After illumination, worms were

593 transplanted back to OP50-seeded NGM plates, allowed to recover for 1-2 days before behavior

594 testing.

\section{Behavioral assays and analysis}

596 Recorded movies, in which worm body centerlines were extracted in real time, were further 597 processed semi-automatically in MATLAB to identify locomotor states (forward locomotion, 598 backward locomotion, pause and turn) and other statistical parameters. We also set up a Graphic 599 User Interface (GUI) that allows human interference and proofreading.

600 Reversal duration was defined as the time from reversal start to reversal end. If one stimulus 601 triggered several reversals, we always scored the first one. Omega turns were identified by either 602 the head touching the body (or tail) or $\theta>135^{\circ}$ within a single head swing (Figure 1 - figure supplement $1 A$ ). The end of a turn was identified when a worm opened its coiled posture and 604 began to move forward.

\section{Transition rate calculation}




$$
\begin{gathered}
r_{i}\left(t_{0}\right) \approx \frac{\int_{\mathrm{t}_{0}-\frac{\Delta \mathrm{t}}{2}}^{\mathrm{t}_{0}+\frac{\Delta \mathrm{t}}{2}} r_{i}(t) d t}{\Delta t} \approx \frac{\int_{t_{0}-\frac{\Delta t}{2}}^{t_{0}+\frac{\Delta t}{2}} p_{i}(t) d t}{\Delta t\left[1-\int_{0}^{\mathrm{t}_{0}-\frac{\Delta \mathrm{t}}{2}}\left(p_{1}(t)+p_{2}(t)\right) d t\right]} \\
=\frac{N_{i}\left(t_{0}-\frac{\Delta t}{2}\right)-N_{i}\left(t_{0}+\frac{\Delta t}{2}\right)}{\Delta t\left[N_{1}\left(t_{0}-\frac{\Delta t}{2}\right)+N_{2}\left(t_{0}-\frac{\Delta t}{2}\right)\right]}
\end{gathered}
$$

609 Here $i=1,2$, represents type-I or type-II transition. $r_{i}(t)$ is the transition rate, $p_{i}(t)$ is the

610 probability density function of the two transitions, $N_{i}(t)$ is the total number of reversals that

611 survive to $t$ for a given transition type, and $\Delta t$ denotes the bin width.

\section{Theoretical account of transition rates and model details}

Detailed description can be found in Appendix.

\section{Quantification and statistical analysis}

615 Quantification and statistical parameters were indicated in the legends of each figure, including the

616 statistical methods, error bars, $n$ numbers (see Supplementary File 4 for more details), and $p$ values.

617 We applied Mann-Whitney U test, $\chi^{2}$ test or Fisher's exact test among samples, two-way ANOVA

618 to determine the significance of difference between groups for two factors, and Kolmogorov-

619 Smirnov test to compare probability distributions from two samples. All multiple comparisons

620 were adjusted using Bonferroni correction. We considered $p$ values of $<0.05$ significant. All

621 analyses were performed using MATLAB.

\section{Data availability}


623 Raw data of calcium imaging experiments and all code used for modeling or figure generation are

624 available for download from https://github.com/Wenlab/Worm-Motor-Sequence-Generation.

625 Source data files have been provided for main figures. 


\section{References}

Abeles, M. (1991). Corticonics: Neural Circuits of the Cerebral Cortex: Cambridge University Press. Alkema, M. J., Hunter-Ensor, M., Ringstad, N., \& Horvitz, H. R. (2005). Tyramine Functions independently of octopamine in the Caenorhabditis elegans nervous system. Neuron, 46(2), 247260. doi:10.1016/j.neuron.2005.02.024

Allen, C., \& Stevens, C. F. (1994). An evaluation of causes for unreliability of synaptic transmission. Proc Natl Acad Sci U S A, 91(22), 10380-10383. doi:10.1073/pnas.91.22.10380

Altun, Z. F., Chen, B., Wang, Z. W., \& Hall, D. H. (2009). High resolution map of Caenorhabditis elegans gap junction proteins. Dev Dyn, 238(8), 1936-1950. doi:10.1002/dvdy.22025

Barnes, T. M., \& Hekimi, S. J. J. o. n. (1997). The Caenorhabditis elegans avermectin resistance and anesthetic response gene unc- 9 encodes a member of a protein family implicated in electrical coupling of excitable cells. 69(6), 2251-2260.

Berg, H. C. (1993). Random Walks in Biology. Princeton, New Jersey.

Berman, G. J., Choi, D. M., Bialek, W., \& Shaevitz, J. W. (2014). Mapping the stereotyped behaviour of freely moving fruit flies. $J R$ Soc Interface, $11(99)$. doi:10.1098/rsif.2014.0672

Bhattacharya, A., Aghayeva, U., Berghoff, E. G., \& Hobert, O. (2019). Plasticity of the Electrical Connectome of C. elegans. Cell, 176(5), 1174-1189 e1116. doi:10.1016/j.cell.2018.12.024

Brenner, S. (1974). The genetics of Caenorhabditis elegans. Genetics, 77(1), 71-94.

Burak, Y., \& Fiete, I. R. (2012). Fundamental limits on persistent activity in networks of noisy neurons. Proc Natl Acad Sci U S A, 109(43), 17645-17650. doi:10.1073/pnas.1117386109

Chalfie, M., Sulston, J. E., White, J. G., Southgate, E., Thomson, J. N., \& Brenner, S. (1985). The neural circuit for touch sensitivity in Caenorhabditis elegans. J Neurosci, 5(4), 956-964.

Costa, W. S., Van der Auwera, P., Glock, C., Liewald, J. F., Bach, M., Schüler, C., . . Bringmann, H. J. N. c. (2019). A GABAergic and peptidergic sleep neuron as a locomotion stop neuron with compartmentalized Ca2+ dynamics. Nature communications, 10(1), 1-15.

Dent, J. A., Davis, M. W., \& Avery, L. J. T. E. j. (1997). avr-15 encodes a chloride channel subunit that mediates inhibitory glutamatergic neurotransmission and ivermectin sensitivity in Caenorhabditis elegans. The EMBO journal, 16(19), 5867-5879.

Dhawale, A. K., Smith, M. A., \& Olveczky, B. P. (2017). The Role of Variability in Motor Learning. Annu Rev Neurosci, 40, 479-498. doi:10.1146/annurev-neuro-072116-031548

Fadok, J. P., Krabbe, S., Markovic, M., Courtin, J., Xu, C., Massi, L., . . Luthi, A. (2017). A competitive inhibitory circuit for selection of active and passive fear responses. Nature, 542(7639), 96-100. doi:10.1038/nature21047

Ganguli, S., Bisley, J. W., Roitman, J. D., Shadlen, M. N., Goldberg, M. E., \& Miller, K. D. (2008). Onedimensional dynamics of attention and decision making in LIP. Neuron, 58(1), 15-25. doi:10.1016/j.neuron.2008.01.038

Gordus, A., Pokala, N., Levy, S., Flavell, S. W., \& Bargmann, C. I. (2015). Feedback from network states generates variability in a probabilistic olfactory circuit. Cell, 161(2), 215-227. doi:10.1016/j.cell.2015.02.018

Gray, J. M., Hill, J. J., \& Bargmann, C. I. (2005). A circuit for navigation in Caenorhabditis elegans. Proc Natl Acad Sci U S A, 102(9), 3184-3191. doi:10.1073/pnas.0409009101

Harvey, C. D., Coen, P., \& Tank, D. W. (2012). Choice-specific sequences in parietal cortex during a virtual-navigation decision task. Nature, 484(7392), 62-68. doi:10.1038/nature10918

Husson, S. J., Liewald, J. F., Schultheis, C., Stirman, J. N., Lu, H., \& Gottschalk, A. (2012). Microbial light-activatable proton pumps as neuronal inhibitors to functionally dissect neuronal networks in C. elegans. PLoS One, 7(7), e40937. doi:10.1371/journal.pone.0040937

Iino, Y., \& Yoshida, K. (2009). Parallel use of two behavioral mechanisms for chemotaxis in Caenorhabditis elegans. J Neurosci, 29(17), 5370-5380. doi:10.1523/JNEUROSCI.3633-08.2009 
674

675

676

677

678

679

680

681

682

683

684

685

686

687

688

689

690

691

692

693

694

695

696

697

698

699

700

701

702

703

704

705

706

707

708

709

710

711

712

713

714

715

716

717

718

719

720

721

722

723

Inagaki, H. K., Inagaki, M., Romani, S., \& Svoboda, K. (2018). Low-Dimensional and Monotonic Preparatory Activity in Mouse Anterior Lateral Motor Cortex. J Neurosci, 38(17), 4163-4185. doi:10.1523/JNEUROSCI.3152-17.2018

Kato, S., Kaplan, H. S., Schrodel, T., Skora, S., Lindsay, T. H., Yemini, E., . . Zimmer, M. (2015). Global brain dynamics embed the motor command sequence of Caenorhabditis elegans. Cell, 163(3), 656669. doi:10.1016/j.cell.2015.09.034

Kawano, T., Po, M. D., Gao, S., Leung, G., Ryu, W. S., \& Zhen, M. (2011). An imbalancing act: gap junctions reduce the backward motor circuit activity to bias $\mathrm{C}$. elegans for forward locomotion. Neuron, 72(4), 572-586. doi:10.1016/j.neuron.2011.09.005

Klaus, A., Alves da Silva, J., \& Costa, R. M. (2019). What, If, and When to Move: Basal Ganglia Circuits and Self-Paced Action Initiation. Annu Rev Neurosci, 42, 459-483. doi:10.1146/annurev-neuro072116-031033

Laurent, P., Soltesz, Z., Nelson, G. M., Chen, C., Arellano-Carbajal, F., Levy, E., \& de Bono, M. (2015). Decoding a neural circuit controlling global animal state in C. elegans. Elife, 4. doi:10.7554/eLife.04241

Leifer, A. M., Fang-Yen, C., Gershow, M., Alkema, M. J., \& Samuel, A. D. (2011). Optogenetic manipulation of neural activity in freely moving Caenorhabditis elegans. Nat Methods, 8(2), 147152. doi:10.1038/nmeth. 1554

Li, Z., Liu, J., Zheng, M., \& Xu, X. Z. (2014). Encoding of both analog- and digital-like behavioral outputs by one C. elegans interneuron. Cell, 159(4), 751-765. doi:10.1016/j.cell.2014.09.056

Lindsay, T. H., Thiele, T. R., \& Lockery, S. R. (2011). Optogenetic analysis of synaptic transmission in the central nervous system of the nematode Caenorhabditis elegans. Nat Commun, 2, 306. doi:10.1038/ncomms 1304

Liu, M., Sharma, A. K., Shaevitz, J. W., \& Leifer, A. M. (2018). Temporal processing and context dependency in Caenorhabditis elegans response to mechanosensation. Elife, 7. doi:10.7554/eLife.36419

Liu, P., Chen, B., Altun, Z. F., Gross, M. J., Shan, A., Schuman, B., . . Wang, Z. W. (2013). Six innexins contribute to electrical coupling of C. elegans body-wall muscle. PLoS One, 8(10), e76877. doi:10.1371/journal.pone.0076877

Long, M. A., Jin, D. Z., \& Fee, M. S. (2010). Support for a synaptic chain model of neuronal sequence generation. Nature, 468(7322), 394-399. doi:10.1038/nature09514

Louie, K., \& Wilson, M. A. (2001). Temporally structured replay of awake hippocampal ensemble activity during rapid eye movement sleep. Neuron, 29(1), 145-156. doi:10.1016/s0896-6273(01)00186-6

Luo, L., Wen, Q., Ren, J., Hendricks, M., Gershow, M., Qin, Y., . . Zhang, Y. (2014). Dynamic encoding of perception, memory, and movement in a C. elegans chemotaxis circuit. Neuron, 82(5), 11151128. doi:10.1016/j.neuron.2014.05.010

Mainen, Z. F., \& Sejnowski, T. J. (1995). Reliability of spike timing in neocortical neurons. Science, 268(5216), 1503-1506. doi:10.1126/science.7770778

Marvin, J. S., Borghuis, B. G., Tian, L., Cichon, J., Harnett, M. T., Akerboom, J., . . Looger, L. L. (2013). An optimized fluorescent probe for visualizing glutamate neurotransmission. Nat Methods, 10(2), 162-170. doi:10.1038/nmeth.2333

Mastrogiuseppe, F., \& Ostojic, S. (2018). Linking Connectivity, Dynamics, and Computations in LowRank Recurrent Neural Networks. Neuron, 99(3), 609-623 e629. doi:10.1016/j.neuron.2018.07.003

Mohammadi, A., Byrne Rodgers, J., Kotera, I., \& Ryu, W. S. (2013). Behavioral response of Caenorhabditis elegans to localized thermal stimuli. BMC Neurosci, 14(1), 66. doi:10.1186/1471-2202-14-66

Mori, I., \& Ohshima, Y. (1995). Neural regulation of thermotaxis in Caenorhabditis elegans. Nature, 376(6538), 344-348. doi:10.1038/376344a0

Nagel, G., Brauner, M., Liewald, J. F., Adeishvili, N., Bamberg, E., \& Gottschalk, A. (2005). Light activation of channelrhodopsin-2 in excitable cells of Caenorhabditis elegans triggers rapid behavioral responses. Curr Biol, 15(24), 2279-2284. doi:10.1016/j.cub.2005.11.032 
Narayan, A., Laurent, G., \& Sternberg, P. W. (2011). Transfer characteristics of a thermosensory synapse in Caenorhabditis elegans. Proc Natl Acad Sci $U$ S A, 108(23), 9667-9672. doi:10.1073/pnas.1106617108

Nguyen, J. P., Shipley, F. B., Linder, A. N., Plummer, G. S., Liu, M., Setru, S. U., . . Leifer, A. M. (2016). Whole-brain calcium imaging with cellular resolution in freely behaving Caenorhabditis elegans. Proc Natl Acad Sci U S A, 113(8), E1074-1081. doi:10.1073/pnas.1507110112

Pierce-Shimomura, J. T., Morse, T. M., \& Lockery, S. R. (1999). The fundamental role of pirouettes in Caenorhabditis elegans chemotaxis. J Neurosci, 19(21), 9557-9569.

Pirri, J. K., McPherson, A. D., Donnelly, J. L., Francis, M. M., \& Alkema, M. J. (2009). A tyramine-gated chloride channel coordinates distinct motor programs of a Caenorhabditis elegans escape response. Neuron, 62(4), 526-538. doi:10.1016/j.neuron.2009.04.013

Pokala, N., Liu, Q., Gordus, A., \& Bargmann, C. I. (2014). Inducible and titratable silencing of Caenorhabditis elegans neurons in vivo with histamine-gated chloride channels. Proc Natl Acad Sci U S A, 111(7), 2770-2775. doi:10.1073/pnas.1400615111

Qi, Y. B., Garren, E. J., Shu, X., Tsien, R. Y., \& Jin, Y. (2012). Photo-inducible cell ablation in Caenorhabditis elegans using the genetically encoded singlet oxygen generating protein miniSOG. Proc Natl Acad Sci U S A, 109(19), 7499-7504. doi:10.1073/pnas.1204096109

Rajan, K., Harvey, C. D., \& Tank, D. W. (2016). Recurrent Network Models of Sequence Generation and Memory. Neuron, 90(1), 128-142. doi:10.1016/j.neuron.2016.02.009

Remington, E. D., Egger, S. W., Narain, D., Wang, J., \& Jazayeri, M. (2018). A Dynamical Systems Perspective on Flexible Motor Timing. Trends Cogn Sci, 22(10), 938-952. doi:10.1016/j.tics.2018.07.010

Remington, E. D., Narain, D., Hosseini, E. A., \& Jazayeri, M. (2018). Flexible Sensorimotor Computations through Rapid Reconfiguration of Cortical Dynamics. Neuron, 98(5), 1005-1019 e1005. doi:10.1016/j.neuron.2018.05.020

Roberts, W. M., Augustine, S. B., Lawton, K. J., Lindsay, T. H., Thiele, T. R., Izquierdo, E. J., .. . Lockery, S. R. (2016). A stochastic neuronal model predicts random search behaviors at multiple spatial scales in C. elegans. Elife, 5. doi:10.7554/eLife.12572

Seeds, A. M., Ravbar, P., Chung, P., Hampel, S., Midgley, F. M., Jr., Mensh, B. D., \& Simpson, J. H. (2014). A suppression hierarchy among competing motor programs drives sequential grooming in Drosophila. Elife, 3, e02951. doi:10.7554/eLife.02951

Serrano-Saiz, E., Poole, R. J., Felton, T., Zhang, F., De La Cruz, E. D., \& Hobert, O. (2013). Modular control of glutamatergic neuronal identity in C. elegans by distinct homeodomain proteins. Cell, 155(3), 659-673. doi:10.1016/j.cell.2013.09.052

Shmiel, T., Drori, R., Shmiel, O., Ben-Shaul, Y., Nadasdy, Z., Shemesh, M., . . Abeles, M. (2006). Temporally precise cortical firing patterns are associated with distinct action segments. $J$ Neurophysiol, 96(5), 2645-2652. doi:10.1152/jn.00798.2005

Skaggs, W. E., \& McNaughton, B. L. (1996). Replay of neuronal firing sequences in rat hippocampus during sleep following spatial experience. Science, 271(5257), 1870-1873. doi:10.1126/science.271.5257.1870

Starich, T. A., Herman, R. K., \& Shaw, J. E. (1993). Molecular and genetic analysis of unc-7, a Caenorhabditis elegans gene required for coordinated locomotion. Genetics, 133(3), 527-541.

Starich, T. A., Xu, J., Skerrett, I. M., Nicholson, B. J., \& Shaw, J. E. J. N. d. (2009). Interactions between innexins UNC-7 and UNC-9 mediate electrical synapse specificity in the Caenorhabditis elegans locomotory nervous system. Neural development, 4(1), 16.

Stephens, G. J., Bueno de Mesquita, M., Ryu, W. S., \& Bialek, W. (2011). Emergence of long timescales and stereotyped behaviors in Caenorhabditis elegans. Proc Natl Acad Sci U S A, 108(18), 72867289. doi:10.1073/pnas.1007868108

Stephens, G. J., Johnson-Kerner, B., Bialek, W., \& Ryu, W. S. (2008). Dimensionality and dynamics in the behavior of C. elegans. PLoS Comput Biol, 4(4), e1000028. doi:10.1371/journal.pcbi.1000028 
Sutton, R. S., \& Barto, A. G. (2017). Reinforcement Learning: An Introduction. . Cambridge, MA: MIT Press.

Tumer, E. C., \& Brainard, M. S. (2007). Performance variability enables adaptive plasticity of 'crystallized' adult birdsong. Nature, 450(7173), 1240-1244. doi:10.1038/nature06390

van Vreeswijk, C., \& Sompolinsky, H. (1996). Chaos in neuronal networks with balanced excitatory and inhibitory activity. Science, 274(5293), 1724-1726. doi:10.1126/science.274.5293.1724

Venkatachalam, V., Ji, N., Wang, X., Clark, C., Mitchell, J. K., Klein, M., . . Samuel, A. D. (2016). Panneuronal imaging in roaming Caenorhabditis elegans. Proc Natl Acad Sci U S A, 113(8), E10821088. doi:10.1073/pnas. 1507109113

White, J. G., Southgate, E., Thomson, J. N., \& Brenner, S. (1986). The structure of the nervous system of the nematode Caenorhabditis elegans. Philos Trans R Soc Lond B Biol Sci, 314(1165), 1-340. doi:10.1098/rstb.1986.0056

Wicks, S. R., Roehrig, C. J., \& Rankin, C. H. (1996). A dynamic network simulation of the nematode tap withdrawal circuit: predictions concerning synaptic function using behavioral criteria. J Neurosci, 16(12), 4017-4031.

Witvliet, D., Mulcahy, B., Mitchell, J. K., Meirovitch, Y., Berger, D. K., Wu, Y., . . Holmyard, D. J. b. (2020). Connectomes across development reveal principles of brain maturation in C. elegans. bioRxiv.

Xu, S., \& Chisholm, A. D. (2016). Highly efficient optogenetic cell ablation in C. elegans using membranetargeted miniSOG. Sci Rep, 6, 21271. doi:10.1038/srep21271

Xu, T., Huo, J., Shao, S., Po, M., Kawano, T., Lu, Y., . . Wen, Q. (2018). Descending pathway facilitates undulatory wave propagation in Caenorhabditis elegans through gap junctions. Proc Natl Acad Sci U S A, 115(19), E4493-E4502. doi:10.1073/pnas.1717022115 


\section{Appendix}

798 This note provides a theoretical account of the time-dependent type-II (RT) transition rate. First, 799 we shall consider how type-II transition arises through a rapid change of neuronal activity, 800 represented experimentally by inter/motor neurons RIV. Second, we shall argue that the 801 introduction of short-term synaptic depression (STD) in the inhibitory synapses may provide an 802 explanation of the type-II transition rate.

\section{A biophysical model}

804 Consider a model neuron (e.g. RIV) in the turning module receiving excitatory inputs through gap 805 junction and inhibitory inputs through synapses (Figure $7 \boldsymbol{A}$ ). The following equation describes the 806 membrane potential $x$ of a neuron determined by the gap junction conductance, inhibitory synaptic 807 conductance and leaky conductance, represented by $G^{\mathrm{g}}, G^{i}$ and $G^{c}$ respectively (Varshney, 808 Chen, Paniagua, Hall, \& Chklovskii, 2011).

$$
C \dot{x}=-G^{g}\left(x-E^{e}\right)-G^{i}\left(x-E^{i}\right)-G^{c}\left(x-E^{c}\right)+I^{\text {Noise }}
$$
of AIB in the first term. $E^{\mathrm{i}}$ and $E^{\mathrm{c}}$ denote the reversal potential of inhibitory synapse and the

812 resting potential. $I^{\text {noise }}$ is Gaussian white noise, which could be regarded as the fluctuation of 813 synaptic currents and other sources of noises that are not explicitly considered in the model. assumption will be relaxed later when we introduce short-term synaptic plasticity in inhibitory 816 synapses, with which $G^{i}$ decreases while $G^{\mathrm{g}}$ and $G^{c}$ remain unchanged. $E^{e}$, the membrane 
817 potential of AIB is treated as a constant, provided that the backward module exhibits persistent

818 activity during a reversal. The fixed point $x_{0}$ is given by

819

$$
x_{0}=\frac{E^{e} G^{g}+E^{i} G^{i}+E^{c} G^{c}}{G^{g}+G^{i}+G^{c}}=\left({ }_{m} E^{m} G^{m}\right) /{ }_{m} G^{m}
$$

820 where $m$ denotes all kinds of inputs, including gap junctions, chemical synapses and leaky channels.

$821 G^{\mathrm{m}}$ and $E^{\mathrm{m}}$ are the conductance of an input and the corresponding target voltage.

822

With small perturbation $x_{0} \rightarrow x_{0}+x$, we make an approximation

$$
C \dot{x}=-\sum_{m} G^{m} \cdot \Delta x
$$

824 provided that the synaptic inputs from other neurons are insensitive to small fluctuation of the 825 membrane potential of a postsynaptic neuron. In this case, the membrane potential can be viewed 826 as a one-dimensional Brownian particle which obeys the following equation

$$
\dot{x}=-k\left(x-x_{0}\right)+\eta, x<x_{t h}
$$

828 where $k=\sum_{m} G^{m} / C$, and $\eta$ is gaussian white noise. To capture the essential process of type-II 829 transition, we assume that the membrane potential $x$ fluctuates around $x_{0}$ during a reversal. Once 830 the noise drives the membrane potential across a threshold $x_{t h}$, the system transitions to a different 831 state: RIV neurons become rapidly depolarized due to a nonlinear rectified activation (Figure $3 \boldsymbol{E}$ 832 and Figure $7 \boldsymbol{B}$ ), starting a turn by activating ventral muscles and terminating the reversal via 833 feedback inhibition (Figure $7 \boldsymbol{A}$ and $7 D$ ).

\section{Calculating the stationary probability density near fixed point with Fokker-Plank equation}


835 Fokker-Planck equation that describes the probability density of the particle position (Hänggi,

836 Talkner, \& Borkovec, 1990) is

$$
\frac{\partial P(x, t)}{\partial t}=\frac{\partial}{\partial x}\left[-k\left(x-x_{0}\right) P(x, t)\right]+\sigma^{2} \frac{\partial^{2} P(x, t)}{\partial x^{2}}
$$

838

840

841 where $\Delta t$ is the time step.

where $\sigma$ determines the amplitude of noise. According to Einstein's theory on Brownian motion, $\sigma$ and the variance of Gaussian white noise $\eta$ has the following relation:

$$
\sigma^{2}=\frac{\Delta t}{2}\left\langle\eta^{2}\right\rangle
$$

The stationary solution for Fokker-Planck equation $\frac{\partial P(x, t)}{\partial t}=0$ is given by:

$$
P(x) \sim e^{-\frac{k\left(x-x_{0}\right)^{2}}{2 \sigma^{2}}}
$$

844 which is similar to Boltzmann distribution, where $\frac{k\left(x-x_{0}\right)^{2}}{2}$ can be viewed as the potential

845 energy $U(x)$, and $\sigma^{2}$ plays the role of temperature $k_{B} T$.

\section{Biophysical understanding of the type-II transition}

847 Now consider a terminal threshold $x_{t h}$, beyond which the system transits to a different state.

848 Assuming that the barrier is high and the particle is restricted in the energy well, we shall next 849 calculate the small, albeit not negligible probability current $J$ crossing the barrier. 


$$
\begin{aligned}
\frac{\partial P(x, t)}{\partial t} & =\frac{\partial}{\partial x}\left[k\left(x-x_{0}\right) P(x, t)\right]+\sigma^{2} \frac{\partial^{2} P(x, t)}{\partial x^{2}} \\
& =\frac{\partial}{\partial x}\left[k\left(x-x_{0}\right) P(x, t)+\sigma^{2} \frac{\partial P(x, t)}{\partial x}\right] \\
& =-\frac{\partial J(x, t)}{\partial x}
\end{aligned}
$$

852 Current $\boldsymbol{J}$ can be rewritten in another form

$$
J(x, t)=-\sigma^{2} e^{-\frac{k\left(x-x_{0}\right)^{2}}{2 \sigma^{2}}} \frac{\partial}{\partial x}\left[e^{\frac{k\left(x-x_{0}\right)^{2}}{2 \sigma^{2}}} P(x, t)\right]
$$

854 Considering a stationary state, we have $\frac{\partial P(x, t)}{\partial t} \approx 0, J \approx$ const

$$
\frac{\partial}{\partial x}\left[e^{\frac{k\left(x-x_{0}\right)^{2}}{2 \sigma^{2}}} P(x)\right]=-\frac{J}{\sigma^{2}} e^{\frac{k\left(x-x_{0}\right)^{2}}{2 \sigma^{2}}}
$$

856

Integrate Eq. (3.3) between $x_{0}$ and $x_{t h}$

$$
\left.\left[e^{\frac{k\left(x-x_{0}\right)^{2}}{2 \sigma^{2}}} P(x)\right]\right|_{x_{0}} ^{x_{\text {th }}}=-\frac{J}{\sigma^{2}} \int_{x_{0}}^{x_{\text {th }}} e^{\frac{k\left(x-x_{0}\right)^{2}}{2 \sigma^{2}}} d x
$$

858 Since the particle is restricted around $x_{0}, P\left(x_{t h}\right) \approx 0$, then we have

$$
J=\frac{\sigma^{2} P\left(x_{0}\right)}{\int_{x_{0}}^{x_{t h}} e^{\frac{k\left(x-x_{0}\right)^{2}}{2 \sigma^{2}}} d x}
$$

861 be 1, and $P(x)=P\left(x_{0}\right) e^{-\frac{k\left(x-x_{0}\right)^{2}}{2 \sigma^{2}}}$ according to Eq. (2.3), we have 


$$
P\left(x_{0}\right) \int_{-\infty}^{+\infty} e^{-\frac{k\left(x-x_{0}\right)^{2}}{2 \sigma^{2}}} d x=1
$$

863 Let $A$ denote $\sqrt{\frac{k}{2 \sigma^{2}}}$

864

$$
P\left(x_{0}\right) \int_{x_{0}-h}^{x_{0}+h} e^{-\frac{k\left(x-x_{0}\right)^{2}}{2 \sigma^{2}}} d x=P\left(x_{0}\right) \int_{-h}^{h} e^{-(A x)^{2}} d x=P\left(x_{0}\right) \frac{\sqrt{\pi}}{A} \operatorname{erf}(A h)=1
$$

865 where $\operatorname{erf}(x)=\frac{2}{\sqrt{\pi}} \int_{0}^{x} \mathrm{e}^{-z^{2}} d z$ is the error function.

By taking the limit $h \rightarrow+\infty, \operatorname{erf}(A h) \rightarrow 1$, we find

$$
P\left(x_{0}\right)=\frac{A}{\sqrt{\pi}}
$$

868 The denominator $\int_{x_{0}}^{x_{\text {th }}} e^{\frac{k\left(x-x_{0}\right)^{2}}{2 \sigma^{2}}} d x$ in Eq. (3.5) can be calculated directly

$$
\int_{x_{0}}^{x_{t h}} e^{\frac{k\left(x-x_{0}\right)^{2}}{2 \sigma^{2}}} d x=\int_{0}^{x_{t h}-x_{0}} e^{(A x)^{2}} d x=\frac{\sqrt{\pi}}{2 A} \operatorname{erfi}\left(A\left(x_{t h}-x_{0}\right)\right)
$$

870 where $\operatorname{erfi}(x)=\frac{2}{\sqrt{\pi}} \int_{0}^{x} \mathrm{e}^{z^{2}} d z$ is imaginary error function

871 Substituting Eqs. (3.8)- (3.9) into Eq. (3.5), we finally obtain the transition rate I

872

$$
r=J=\frac{k}{\pi} \cdot \operatorname{erfi}^{-1}\left(\sqrt{\frac{k}{2 \sigma^{2}}}\left(x_{t h}-x_{0}\right)\right)
$$


874 Since the membrane time constant of a neuron $(\sim 10 \mathrm{~ms})$ is much smaller than the behavioral

875 timescale $(\sim \mathrm{s})$, the probability density approaches the stationary state rapidly and Eq. (3.10) is a

876 constant rather than a function of time, resulting in an exponential distribution of the dwell time.

877 To explain the time-dependent type-II transition rate (Figure $\mathbf{1 C}$-D), we introduce plasticity in the

878 inhibitory synapses, analogous to short-term synaptic depression (STD).

As the inhibition onto the model neuron becomes weaker, fixed point $x_{0}$ moves towards

threshold $x_{t h} ; \quad k$, which characterizes the steepness of the potential energy, becomes shallower.

881 Both analytical calculation and simulation suggest that the change of $x_{0}$ has a much larger effect

$$
\begin{aligned}
& r_{2}(t)=\frac{k}{\operatorname{xerfi}} i^{1}\left(\sqrt{\frac{k}{2^{2}}}\left(x_{t h} \quad x_{0}(t)\right)\right) \\
& =\frac{k}{-} \times e r f i{ }^{1}\left(\sqrt{\frac{k}{2^{2}}}\left(x_{t h} x_{f i n}+\left(x_{f i n} \quad x_{i n i}\right) e^{(t /)}\right)\right) \\
& =k_{1} \times \operatorname{erfi}{ }^{1}\left(k_{2}+k_{3} e^{(t /)}\right)
\end{aligned}
$$

Eq. (4.1) was used to fit the type-II transition rate.

\section{References}

Hänggi, P., Talkner, P., \& Borkovec, M. (1990). Reaction-rate theory: fifty years after Kramers. Reviews of Modern Physics, 62(2), 251-341. doi:10.1103/RevModPhys.62.251 properties of the Caenorhabditis elegans neuronal network. PLoS Comput Biol, 7(2), e1001066. doi:10.1371/journal.pcbi.1001066 


\section{Figure legends}

Figure 1. Flexible motor sequence generation during $C$. elegans escape responses. (A) Optogenetic stimulation of mechanosensory neurons ALM/AVM (Pmec-4::ChR2) was carried out with blue light $\left(473 \mathrm{~nm}, 4.63 \mathrm{~mW} / \mathrm{mm}^{2}\right)$ for $1.5 \mathrm{~s}$ during animal forward movement. Identical stimuli could induce four different behavioral outputs: reversal followed by an omega turn, reversal followed by forward movement, pause state or forward movement (no response). (B) Definition of type-I and type-II transitions. (C) Reversal length distribution (left) and transition rates (right) during ALM/AVM (Pmec-4::ChR2) triggered escape responses. Total number of trials, $n=674$; reversal without turn, $n=272$; reversal with turn, $n=322$; no response, $n=79$; pause, $n=1$. Here and below, the error bars of transition rates represent 95\% binomial proportion confidence interval. (D) Reversal length distribution (left) and transition rates (right) during thermal stimulus induced escape responses. Wild-type, $n=385$. Animal heads were thermally stimulated by an infrared laser (1480 nm; $400 \mathrm{~mA}$ ) for $0.75 \mathrm{~s}$. Solid lines are theoretical fits using Eq. (4) and fitted type-II transition rates pass goodness-of-fit test $(p>0.05)$.

The online version of this article includes the following figure supplement(s) for figure 1:

Figure 1-figure supplement 1. Definition of the type II transition and statistical test of reversal length distribution.

Figure 2. Interneurons AIB are crucial for motor state transitions. (A) Simplified circuit diagram underlying the gentle touch induced escape responses. Neurons were grouped into four modules based on their functional roles and activity patterns. (B) Calcium activity of AIB during 
spontaneous reversals before type-I $(n=23)$ and type-II $(n=36)$ transitions in unrestrained behaving animals (Pinx-1::GCaMP6;Pinx-1::wCherry). Here, data are aligned to the ends of reversals (vertical dashed line, $t=0)$. Heat map across trials (up) and $\Delta R(t) / R_{0}($ Mean $+/-$ SEM, bottom) are shown. (C) Ramping rate of calcium activity in AIB. Up, raw single trial $\Delta R(t) / R_{0}$ from reversal start to reversal end. The ramping rate is the slope of the red line, fitted by linear regression. Bottom, ramping rates of AIB during type-I and type-II transitions. Each color (Mean \pm SEM) represents single animal data across multiple trials. Total 9 animals (Pinx-1::GCaMP6;Pinx-1::wCherry) were tested. Very short reversals (less than $1.5 \mathrm{~s}$ ) are excluded, for some of them have negative ramping rates and the slope estimate is susceptible to noise (but including those trials doesn't affect our conclusion). $* * p<0.01$, two-way ANOVA. (D) Optogenetic activation of AIB (635 nm, 4.46 $\left.\mathrm{mW} / \mathrm{mm}^{2}, 7 \mathrm{~s}\right)$ or inhibition of AIB $\left(561 \mathrm{~nm}, 21.71 \mathrm{~mW} / \mathrm{mm}^{2}, 12 \mathrm{~s}\right)$ during ALM/AVM (473 nm, $14.71 \mathrm{~mW} / \mathrm{mm}^{2}, 1.5 \mathrm{~s}$ ) triggered avoidance behaviors, reversal durations (bar graph) and fractions of animals executing omega turns (pie chart) are shown. Error bars are SEMs. Bar graph, MannWhitney U test. Pie chart, $\chi^{2}$ test. $* p<0.05$, *** $p<0.001$, **** $p<0.0001$. Here and below, the actual turning percentages $\left(n_{\text {turn }} / n_{\text {total }}\right)$ are noted beside the pie chart and numbers within the bars indicate trial number $n$. (E-F) Reversal length distribution (E) and transition rates (F) during escape responses when AIB were persistently hyperpolarized through an exogenous expression of the potassium channel TWK-18. Control group is from Figure 1C.

The online version of this article includes the following figure supplement(s) for figure 2:

Figure 2-figure supplement 1. Local interneurons AIB and RIM in the backward module differentially modulate motor state transitions. 
Figure 3. Feedforward excitation via electrical coupling drives omega turns. (A) Calcium activity of RIV during the type-I $(n=38)$ and the type-II $(n=18)$ transitions in spontaneous behaving animals (Plim-4::GCaMP6). Here, data are aligned to the ends of reversals (vertical dashed line, $t=0$ ). Heat map across trials (left) and $\Delta$ ? $(t)$ (Mean +/- SEM, right) are shown. (B) Optical neurophysiology for probing the feedforward coupling between the backward module and the turning module. (C) Simultaneous optogenetic activation of AIB $\left(635 \mathrm{~nm}, 6.11 \mathrm{~mW} / \mathrm{mm}^{2}\right)$ and calcium imaging of RIV in immobilized animals. $\Delta R(t) / R_{0}$ (Mean $\left.\pm \mathrm{SEM}\right)$ under different genetic backgrounds are shown: control (ATR) is wild-type (dark blue); inx-1 unc-9 unc-7 triple mutant (red); calcium imaging of RIV in the presence of moderate inhibition $\left(561 \mathrm{~nm}, 1.94 \mathrm{~mW} / \mathrm{mm}^{2}\right)$ of 947 RIV (light blue, Plim-4::Arch). The control (no ATR) represents imaging data from wild-type 948 animals without feeding all-trans retinal (grey). ${ }^{* * * *} p<0.0001$, two-way ANOVA. (D) Probability of a reversal followed by a turn (left) and type-II transition rates (right) for gap junction deficient mutants. AIB, expressing Chrimson, were optogenetically activated for $7 \mathrm{~s}(635 \mathrm{~nm}, 4.46$ $\mathrm{mW} / \mathrm{mm}^{2}$ ) in wild-type animals (green), gap junction deficient mutants inx-1 (red), and inx-1 mutants, in which INX-1 channels were restored specifically in AIB (blue). Error bars indicate $95 \%$ binomial proportion confidence interval. $\chi^{2}$ test. $* * * * p<0.0001$. (E) Simultaneous optogenetic 954 activation of AIB $\left(635 \mathrm{~nm}, 6.11 \mathrm{~mW} / \mathrm{mm}^{2}\right)$ and calcium imaging of RIV in unrestrained behaving 955 animals. Left, calcium activity heatmap across trials. $t<0$ represents reversals. Omega turns start 956 at $t=0$. Right, $\Delta$ ? $(t)$ (mean \pm SEM) are shown (blue). (F) Data are related to Figure $3 \mathbf{E}$, but $t=0$ 957 is aligned to the beginning of AIB stimulation.

958 The online version of this article includes the following figure supplement(s) for figure 3: 
959 Figure 3-figure supplement 1. Optogenetic activation of AIB and calcium imaging of RIV in

960 different experimental conditions.

961 Figure 3-figure supplement 2. Possible polysynaptic pathways from AIB to RIV (less than four layers are shown) according to C. elegans connectome.

Figure 4. Glutamatergic inhibition modulates type-II transition. (A) Reversal durations (left) and type-II transition rates (right) in glutamatergic synaptic transmission deficient animals upon strong and persistent optogenetic activation of AIB. Optogenetic stimulation was delivered for $7 \mathrm{~s}$ using red light $\left(635 \mathrm{~nm}, 4.46 \mathrm{~mW} / \mathrm{mm}^{2}\right)$. Right, compare $r 2$ in eat-4 mutant or in Pnpr-9::TeTx with control animals across the whole distribution (Kolmogorov-Smirnov test, $p=6.2 \mathrm{e}-7, p=0.0026$ ) or within a time window $\left(\chi^{2}\right.$ test: $* p<0.05, * * p<0.01, * * * p<0.001$, **** $\left.p<0.0001\right)$. (B) Reversal durations (left) and type-II transition rates (right) in $\mathrm{GluCl}$ receptor mutants upon optogenetic 971 activation of AIB $\left(635 \mathrm{~nm}, 4.46 \mathrm{~mW} / \mathrm{mm}^{2}\right)$. Right, compare $r_{2}$ in triple receptor mutant with 972 control animals across the whole distribution (Kolmogorov-Smirnov test, $p=5.9 \mathrm{e}-8$ ) or within a 973 time window $\left(\chi^{2}\right.$ test: $\left.* p<0.05, * * p<0.01\right)$. (C) Genes encoding GluCl receptors were expressed 974 in local interneurons RIB and AIY respectively. GFP reporter lines were constructed using avr-14, $975 a v r-15$ and $g l c-1$ promoters, respectively; mCherry (or wCherry) reporters were used for cell 976 identification. Expression pattern from one section is showed. Scale bar, $10 \mu \mathrm{m}$. (D) Simultaneous 977 optogenetic activation of AIB $\left(635 \mathrm{~nm}, 6.11 \mathrm{~mW} / \mathrm{mm}^{2}\right)$ and calcium imaging of RIB in 978 immobilized animals under wide-type (control (ATR)) (blue) or eat-4 glutamate deficient mutant 979 background (red). $\Delta R(t) / R_{0}(\mathrm{Mean} \pm \mathrm{SEM})$ are shown. $t=0$ represents the beginning of AIB 980 stimulation. The control group (no ATR) (grey) represents imaging data from animals without 
981 feeding all-trans retinal. $* * * * p<0.0001$, two-way ANOVA. (E) Functional coupling between AIB 982 and RIB neurons was directly tested through glutamate imaging. Upon stimulation of AIB (Pnpr983 9::Chrimson), the process (red), cell body (green) of the RIB (Psto-3::iGluSnFR) and the RIB 984 process in eat-4 glutamate deficient mutants (grey) exhibited distinct fluorescence signals. The 985 control group (blue) represents imaging data from animals without feeding all-trans retinal. Raw 986 iGluSnFR imaging was recorded at $150 \mathrm{~Hz}$. Trial average (bold color) and SEM (shaded region) 987 are shown. Inset, the fluorescence signal of the process (red) is fitted with a double exponential 988 function (blue), $B\left(e^{-\frac{t}{\tau_{1}}}-e^{-\frac{t}{\tau_{2}}}\right)+c$, where $B, \tau_{1}, \tau_{2}$ and $c$ are free parameters. $\tau_{1}$ represents 989 the time constant of glutamate signal decay, and $c$ is the baseline constant, which was later 990 subtracted in the inset plot.

991 For reversal duration, Error bars indicate SEM and Mann-Whitney U test. *** $p<0.001$, **** 992 $p<0.0001$. All multiple comparisons were adjusted using Bonferroni correction.

993 The online version of this article includes the following figure supplement(s) for figure 4 :

994 Figure 4-figure supplement 1. Glutamatergic inhibitions between AIB and RIB flexibly control 995 the motor state transitions.

996 Figure 4-figure supplement 2. Functional coupling between AIB and RIB neurons was directly 997 tested through glutamate imaging. Figure 5. Local interneurons RIB promote both turning and forward behaviors. (A) Calcium 1000 activity of RIB during type-I (up, $n=41$ ) and type-II transitions (bottom, $n=25) . \Delta$ ? $(t)$ (mean \pm 1001 SEM) are shown. $t=0$ is aligned to the initiation of forward movement (up) or an omega turn 
1002 (bottom). (B) Statistics of motor state transitions (left) and representative curvature kymographs 1003 (right) upon optogenetic manipulation of RIB. Left, the probability for a transition and its 95\% 1004 confidence limits were computed. RIB::Chrimson ${ }^{1}, n=65$, red light $\left(635 \mathrm{~nm}, 3.75 \mathrm{~mW} / \mathrm{mm}^{2}\right)$; 1005 RIB::Chrimson ${ }^{2}, n=82$, red light $\left(635 \mathrm{~nm}, 1.00 \mathrm{~mW} / \mathrm{mm}^{2}\right)$; RIB::Arch, $n=83$, green light $(561 \mathrm{~nm}$, $\left.10068.14 \mathrm{~mW} / \mathrm{mm}^{2}\right)$. Right, animals crawled on fresh agar plates. Body curvature was normalized by a $1007 k \cdot L$, where $L$ is the body length. Green (or red) shaded regions show selected spatiotemporal 1008 regions for optogenetic inhibition (or activation). The kymograph of turning behaviors exhibits 1009 longer cycles to complete body bending and larger body curvature, which are different from those 1010 during forward movements. (C) Reversal length distributions (left) and transition rates (right) 1011 when ALM/AVM activation was followed by optogenetic inhibition of RIB (12 s green light, 561 $1012 \mathrm{~nm}, 1.94 \mathrm{~mW} / \mathrm{mm}^{2}$ ). Pmec-4::ChR2;Psto-3::Arch, $n=173$. Control group is from Figure 1C. (D) 1013 Calcium imaging of RIV in wild-type (blue) and RIB-ablated animals (orange) upon optogenetic 1014 stimulation of AIB in freely behaving animals. $\Delta R(t) / R_{0}$ (Mean \pm SEM) are shown. (E) Data are 1015 related to Figure 5D, but all trials are aligned to the onset of an omega turn. (F) Reversal length 1016 during ALM/AVM-triggered escape responses in RIB-ablated animals or in animals where 1017 chemical synaptic transmission from RIB was blocked. Error bars indicate SEM. Mann-Whitney $1018 \mathrm{U}$ test, $* * * * p<0.0001$. All multiple comparisons were adjusted using Bonferroni correction.

1019 The online version of this article includes the following figure supplement(s) for figure 5:

1020 Figure 5-figure supplement 1. RIB receive feedforward inhibition to modulate type-II transition 1021 and neurons in the turning module might contribute to reversal termination. 
1023 Figure 6. Feedback inhibitions from the turning module contribute to reversal termination. (A) 1024 Expression pattern of Plim-4::Arch::GFP. Scale bar, $10 \mu \mathrm{m}$. (B) Left, escape responses induced by 1025 optogenetic activation of AIB $\left(473 \mathrm{~nm}, 14.71 \mathrm{~mW} / \mathrm{mm}^{2}, 1.5 \mathrm{~s}\right)$ or thermal stimulus $(1480 \mathrm{~nm}, 0.75$ $1026 \mathrm{~s})$ followed by optogenetic inhibition of Arch-expressed interneurons $\left(561 \mathrm{~nm}, 21.71 \mathrm{~mW} / \mathrm{mm}^{2}, 12\right.$ 1027 s). Bar graph, reversal durations, Mann-Whitney U test and error bars are SEMs. Pie chart, 1028 fractions of trials executing omega turns, $\chi^{2}$ test. * $p<0.05$, *** $p<0.001, * * * * p<0.0001$. All 1029 multiple comparisons were adjusted using Bonferroni correction. Right, schematic diagram for 1030 selective inhibition of RIV near animal head during thermally induced (1480 nm, $0.75 \mathrm{~s})$ escape 1031 responses. (C) Up, reversal length distribution (left) and transition rate (right) in RIV/SAAD/SMB 1032 ablated animals. Bottom, spontaneous run length distribution (left) and transition rate from forward 1033 to backward movements (right) in wild-type animals. Insets are semiology plot of survival 1034 functions: proportion of trials moving backward (up) or forward (bottom) until $t$. We considered 1035 only trials with reversal duration $\geq 3 \mathrm{sec}$ and forward duration $\geq 2 \mathrm{sec}$.

Figure 7. A model summary for motor sequence generation. (A) Schematics of neuronal circuit of C. elegans avoidance behavior. Feedforward excitations between local interneurons AIB and RIV trigger turning behavior. Glutamatergic inhibitions between AIB and RIB flexibly control the motor state transitions. Feedback inhibitions from the turning module help terminate backward

1041 behavior. (B) Illustration of the biophysical meaning of the type-II transition. The sub-threshold 1042 membrane potential of RIV fluctuates around the fixed point $x_{0}$, just like a particle (red) in an 1043 energy well. When RIV membrane potential crosses the threshold $x_{t h}$, RIV along with other 1044 neurons in the turning module become fully activated and a turn starts. (C) A simple stochastic 1045 attractor model without short-term synaptic plasticity cannot account for the experimentally 
1046 observed transition rate. (D) Schematics of a three-module model based on the animal connectome 1047 and experimentally identified functional motifs. Short-term synaptic depression (STD) was 1048 introduced in the feedforward inhibition. (E) Type-II transition rate during ALM/AVM triggered 1049 escape responses. Pink line is a theoretical fit using Eq. (4) and orange line is a fit using standard 1050 sigmoid function, both of which have three free parameters. $\tau_{g}$ in Eq. (4) is given by the 1051 glutamate decay time constant in Figure $\mathbf{4 E}$, while $\tau$ in the standard sigmoid function is a free 1052 parameter. 


\section{Supplementary Figure legends}

1055

1056

1057

1058

1059

1060

1061

1062

1063

1064

1065

1066

1067

1068

1069

1070

1071

1072

1073

1074

Figure 1-figure supplement 1. Definition of the type II transition and statistical test of reversal length distribution. (A) Left, examples of reorientation angle $\theta$ during the type-I (up) and the typeII transition (bottom). $\theta$ was measured as the angle between the head vector (BA) and the tail vector (DC). The worm body centerline was equally divided into 100 segments. The head vector (BA) is denoted as point B (mean coordinate of the 10th-14th segment) to A (mean coordinate of the 1 st-5th segment), while the tail vector is denoted as point D (mean coordinate of 96th-100th segment) to C (mean coordinate of the 86th-90th segment). Immediately after the end of reversal, we identified a frame in which the worm exhibited a highly coiled body posture to calculate $\theta$. If the head touched the body or tail, $\theta$ was computed from the frame before touching. Right, distribution of $\theta$ during ALM/AVM (Pmec-4::ChR2) triggered escape responses. A type-II transition was identified by either the head touching the body (or tail) or $\theta>135^{\circ}$ within a single head swing. Total trials, $n=594$; type-I transition, $n=272$; type-II transition (touching the body or tail), $n=283$; type-II transition (no touching), $n=39$. (B) A Gaussian mixture model to fit the reversal length distribution during ALM/AVM (Pmec-4::ChR2) triggered escape responses. We performed the likelihood ratio test between the unimodal Gaussian distribution fit and the bimodal Gaussian mixture model fit. After computing the difference of the negative log likelihoods, we found a $p$-value $=5.4 \mathrm{e}-5$ using the $\chi_{k}^{2}$ distribution, where $k=3$ is the difference of the number of free parameters in the unimodal (total 2 parameters) and bimodal (total 5 parameters) distribution. At level $\alpha=0.01$, we have sufficient evidence that the bimodal distribution fits the reversal length better than the unimodal distribution. 
1076 Figure 2-figure supplement 1. Local interneurons AIB and RIM in the backward module

1077 differentially modulate motor state transitions. (A) The schematic diagram of C. elegans nervous 1078 system. (B) Multi-neuron calcium imaging in freely behaving animals (Pnmr-1/ Plim-4/ Plgc-55/ 1079 Pnpr-9::NLS::wCherry::SL2::GCaMP6). Interneurons in the backward and forward modules were 1080 simultaneously imaged using a confocal microscopy (see Materials and Methods for details). Data 1081 are aligned to the onset of a reversal (up, vertical dashed lines, $t=0$ ) or the end of a turn (bottom, 1082 vertical dashed lines, $t=0)$. Heat map across trials and $\Delta R(t) / R_{0}($ Mean $+/$ - SEM) across trials are 1083 shown. (C) Ramping rate of AIB calcium activity during thermally-induced escape responses. 1084 Ramping rate is significantly higher in type-II transition (9 animals, each point has more than 3 1085 trials). $* p<0.05$, two-way ANOVA. (D-E) Characterization of the probability of type-II transition 1086 during optogenetic stimulation of two different interneurons AIB (left) and RIM (right) for varying 1087 durations. Longer activation of AIB consistently led to a higher probability of type-II transition 1088 (left). The control groups are animals performing spontaneous motor state transitions without 1089 optogenetic stimulation. A blue laser $\left(473 \mathrm{~nm}, 14.71 \mathrm{~mW} / \mathrm{mm}^{2}\right)$ was used to stimulate ChR2 1090 expressed neurons. (F-G) Hyperpolarization of RIM (Ptdc-1::Arch) or AIB (Pnpr-9::Arch) both 1091 affected reversal duration (bar graph), but only inhibiting AIB affected the probability of type-II 1092 transition (pie chart). In F, simultaneous optogenetic activation of AIB (or RIM) (635nm, 4.46 $1093 \mathrm{~mW} / \mathrm{mm}^{2}$ for AIB or $473 \mathrm{~nm}, 14.71 \mathrm{~mW} / \mathrm{mm}^{2}$ for RIM) and inhibition $\left(561 \mathrm{~nm}, 6.80 \mathrm{~mW} / \mathrm{mm}^{2}\right)$ of 1094 RIM (or AIB) were carried out for 7 (or 5) seconds. In G, RIM were activated for varying durations, 1095 while AIB were inactivated through an exogenous expression of the potassium channel TWK-18. 1096 (H-I) Reversal length distribution and transition rates after optogenetic ablation of AIB. 1097 ALM/AVM were optogenetically activated for 1.5 seconds to trigger avoidance behavior. AIB 
1098 were ablated by exogenous expression of a membrane targeted PH-miniSOG. Data from Figure

$10991 \mathbf{C}$ are used as control.

1100 For reversal duration, error bars indicate SEMs. Mann-Whitney U test. For probability of turn,

1101 error bars indicate $95 \%$ binomial proportion confidence interval. $\chi^{2}$ test. $* p<0.05, * * p<0.01$, ***

$1102 p<0.001, * * * * p<0.0001$. All multiple comparisons were adjusted using Bonferroni correction.

Figure 3-figure supplement 1. Optogenetic activation of AIB and calcium imaging of RIV in

1107 during thermal stimulus induced escape responses in freely moving animals. $\Delta$ ? $(t)=[\Delta R(t)-$

$1108 \Delta R(t=0)] / R_{0}$ (mean $\left.\pm \mathrm{SEM}\right)$ are shown. $t=0$ is aligned to the initiation (up) or termination (bottom) 1109 of an omega turn.

1111 Figure 3-figure supplement 2. Possible polysynaptic pathways from AIB to RIV (less than four

1112 layers are shown) according to C. elegans connectome. SDQ were oxygen-sensing neurons and 1113 topological cluster analyses suggest ADA may be involved in chemosensation. RIM, RIS and RIV 1114 have direct electrical coupling with AIB. 
1118 AIB, RIV and RIB are highlighted. (B) Reversal length during optogenetic activation of AIB in 1119 various glutamate postsynaptic receptor mutants and restoring eat-4 specifically in AIB in different 1120 eat-4 glutamate deficient mutants. GluCl single and double mutants exhibited longer reversals than 1121 the triple mutants (Figure 4B), suggesting that AVR-14, AVR-15 and GLC-1 proteins function 1122 synergistically to mediate feedforward inhibition. Error bars indicate SEMs. * $p<0.05$, ** $p<0.01$, $1123 * * * * p<0.0001$. Mann-Whitney U test. All multiple comparisons were adjusted using Bonferroni 1124 correction. (C) Single trial data related to Figure 4D.

Figure 4-figure supplement 2. Functional coupling between AIB and RIB neurons was directly

1127 tested through glutamate imaging. (A) Single trial data of the glutamatergic signal on the process 1128 of RIB after AIB activation onset, related to Figure 4E. Raw data and smoothed data (bold line) 1129 are showed. (B) Examination of AIB calcium activity after optogenetic activation of AIB, related 1130 to Figure 4E. Raw data and smoothed data (bold line) are showed.

Figure 5-figure supplement 1. RIB receive feedforward inhibition to modulate type-II transition

1133 and neurons in the turning module might contribute to reversal termination. (A) Single trial data 1134 related to Figure 5A. Dashed lines indicate the initiation of forward movement (up) or omega turns 1135 (bottom). (B) Persistent inhibition of RIB by expressing an exogenous histamine channel (HisCl) 1136 during ALM/AVM-triggered escape responses. Type-II transition quickly approached a plateau at 1137 a lower rate, which is consistent with our model prediction and Figure 5C. Control group is from 1138 Figure 1C. (C) Single trial data related to Figure 5D-E. (D) Left, simultaneous optogenetic 1139 activation of AIB and calcium imaging of RIV or SAAD/SMB (Pnpr-9::Chrimson;Plim- 
$11404:: \mathrm{GCaMP} 6)$ in freely behaving animals. $t=0$ is aligned to the onset of an omega turn. $\Delta$ a $(t)$ (mean

$1141 \pm$ SEM) are shown. Right, smoothed single trial data. Dashed lines indicate the start of a turn. 
1144 Note that all videos were played at $0.625 \times$ speed. Blue/red light on indicates optogenetic excitation

1145 using blue/red light, while green light on indicates optogenetic inhibition using green light.

1146 Video 1. Three main types of escape responses, Related to Figure 1.

1147 Optogenetic activation of ALM/AVM mechanosensory neurons in freely moving worms for 1.5 1148 seconds induced three types of responses.

1149 Video 2. AIB neurons are crucial for turn and backward movement, Related to Figure 2 and 1150 Figure 2-figure supplement 1.

1151 Optogenetic activation of AIB neurons in free-moving animals reliably triggered backward 1152 movement followed by omega turns. Inhibition of AIB neurons quickly terminated backward 1153 movement.

1154 Video 3. RIV neurons are essential for omega turns, Related to Figure 6.

1155 Optogenetic ablation of RIV/SAA/SMB neurons abolished type-II transition, and animals could 1156 no longer generate a complete turn but executed a much longer reversal.

1157 Video 4. Optogenetic activation of AIB under various genetic backgrounds, Related to Figure $1158 \quad 3$ and Figure 4.

1159 Optogenetic activation of AIB in eat-4 glutamate vesicular transport deficient mutants or 1160 glutamate postsynaptic receptor mutants induced an omega turn preceded by a much shorter 1161 reversal. Specifically restoring eat-4 in AIB or glc-1 in AIY promoted a longer reversal. Activating 1162 AIB in gap junction deficient mutant inx-1 reduced the probability of omega turn, which were 1163 rescued by restoring inx-1 in AIB. 
1164 Video 5. Optogenetic manipulation of RIB can induce motor state transitions, Related to 1165 Figure 5.

1166 Strong activation of RIB during forward movement reliably triggered turns; while inhibiting RIB 1167 during forward movement led to a pause state.

1168 Video 6. Optogenetic inhibition of RIB or RIV/SAA/SMB neurons during escape responses 1169 prolonged reversal durations, Related to Figure 5 and Figure 6. 


\section{Supplementary Files}

1172 Supplementary File 1. Strains information

1173 Supplementary File 2. Associated plasmids information

1174 Supplementary File 3. Associated promoter and gene information

1175 Supplementary File 4. Sample size and replicate number for all experiments 


\section{Source data}

$1178 \quad$ Figure 1-source data 1

1179 Source data for Figure 1 and Figure 1—figure supplement 1.

1180 Figure 2-source data 1

1181 Source data for Figure 2 and Figure 2-figure supplement 1.

1182 Figure 3-source data 1

1183 Source data for Figure 3.

1184 Figure 4-source data 1

1185 Source data for Figure 4 and Figure 4-figure supplement 1-2.

1186 Figure 5-source data 1

1187 Source data for Figure 5 and Figure 5-figure supplement 1.

$1188 \quad$ Figure 6-source data 1

1189 Source data for Figure 6. 
A
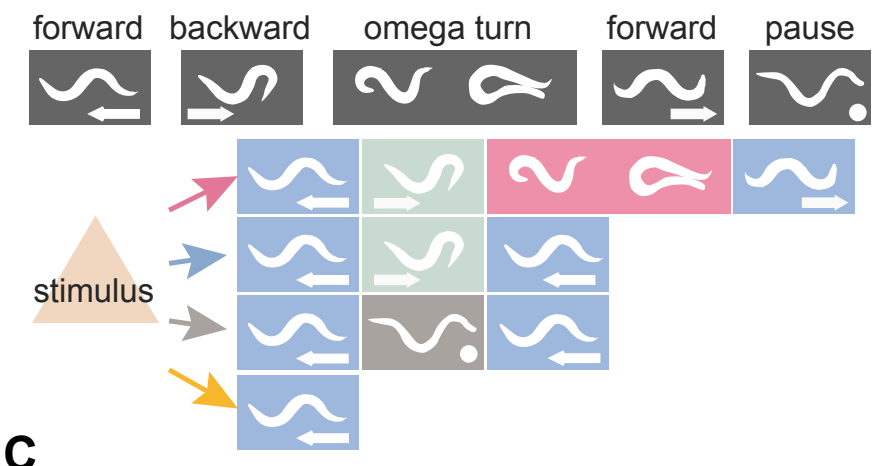

C
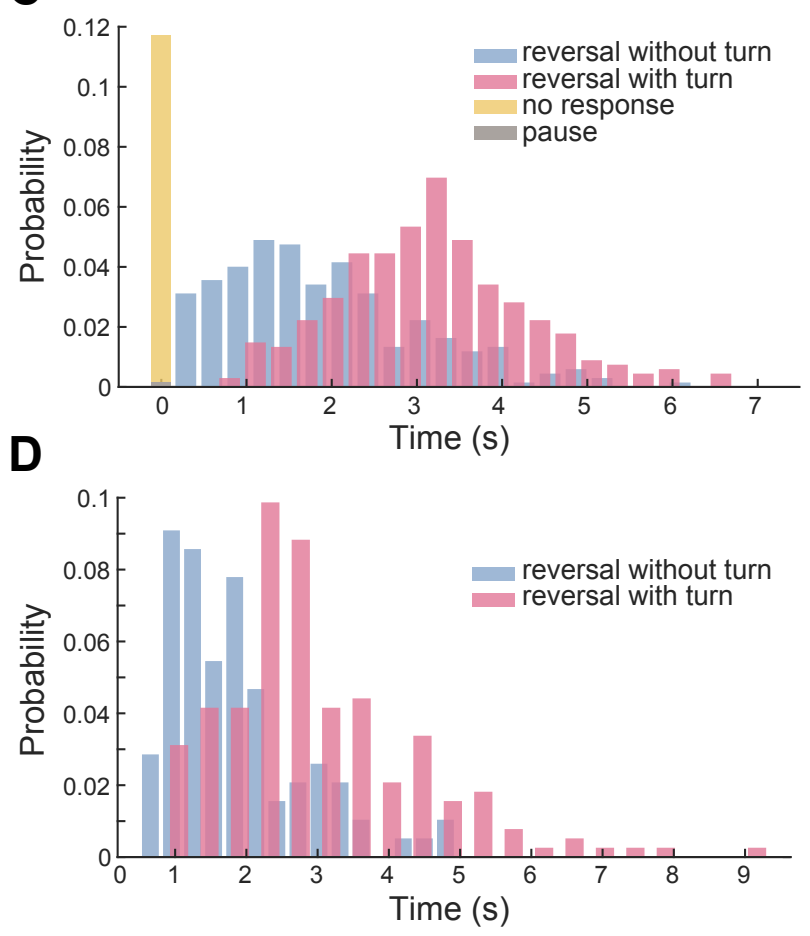

B

Gentle touch
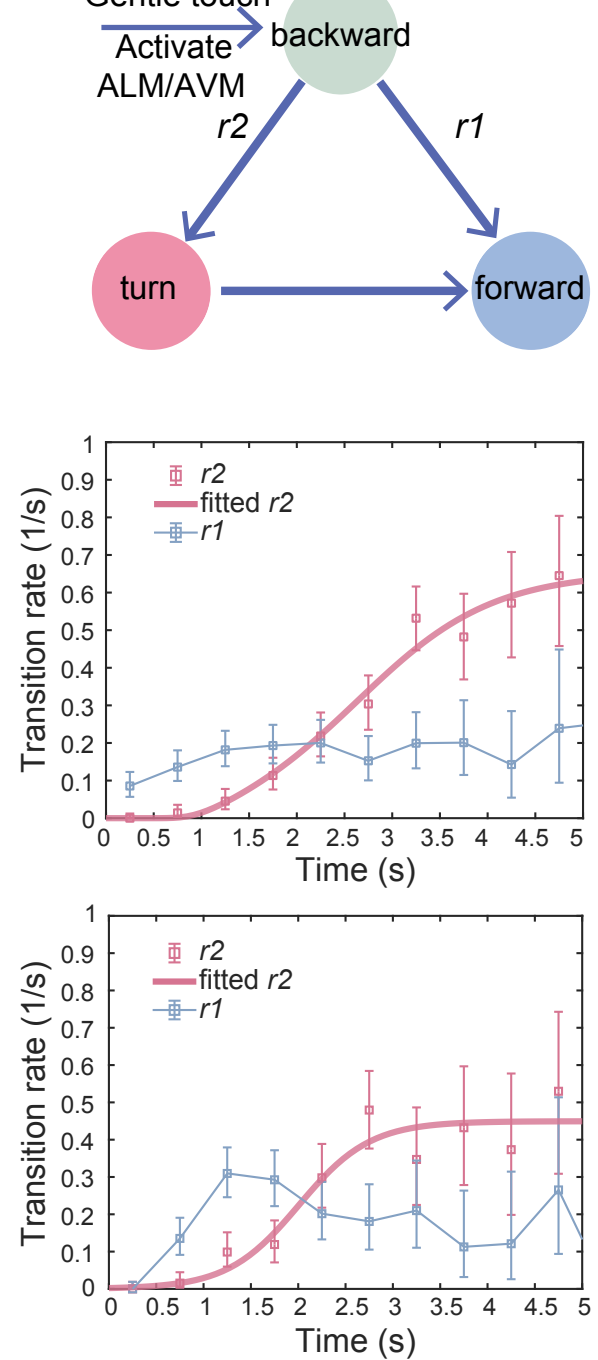
A

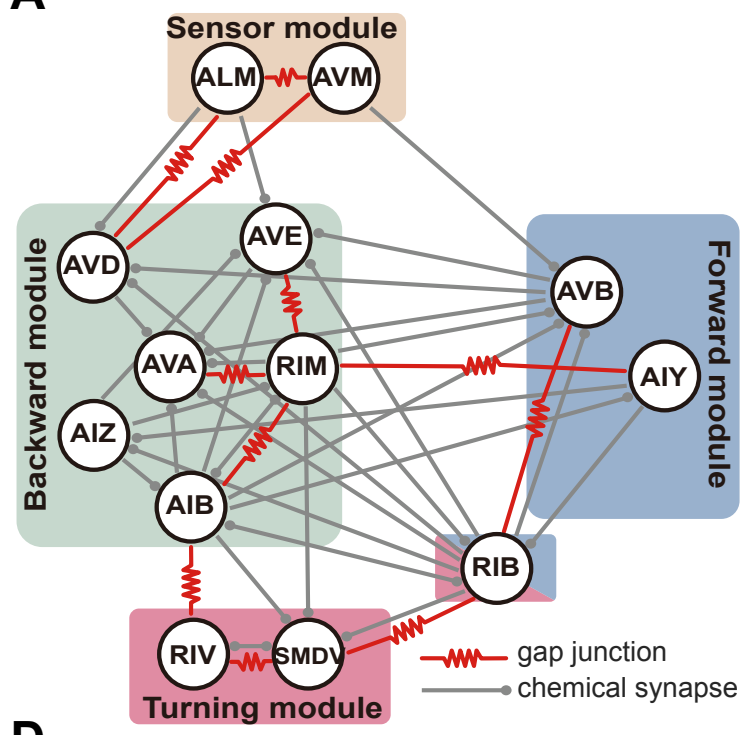

D

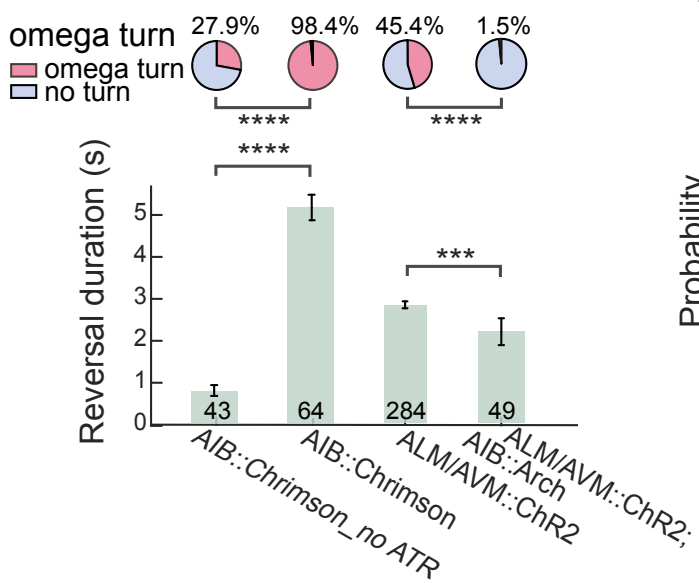

B
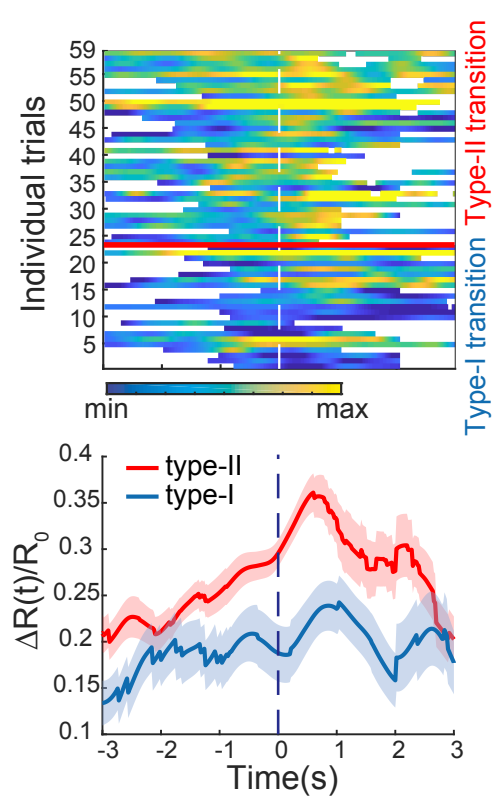

E

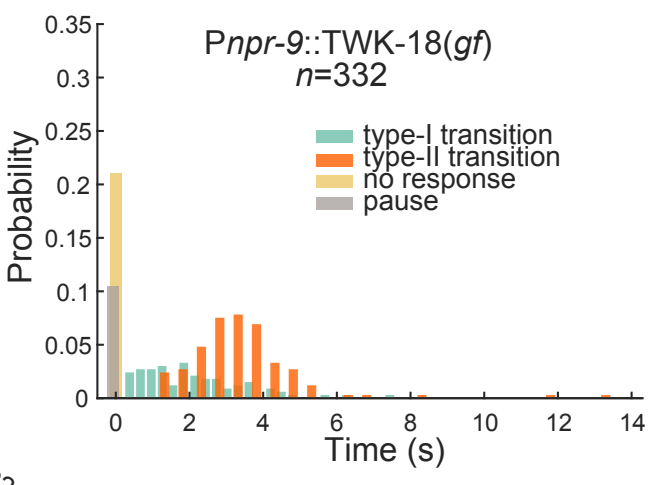

Ramping rate $\zeta=\mathrm{d} \mathcal{R} / \mathrm{d} t$

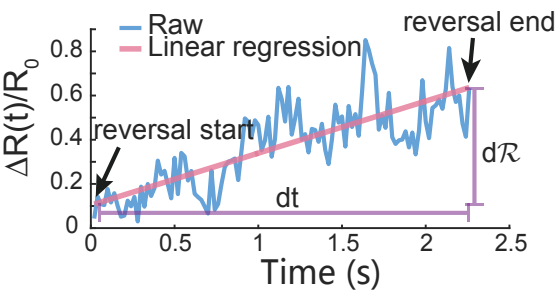

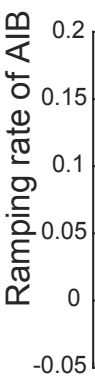

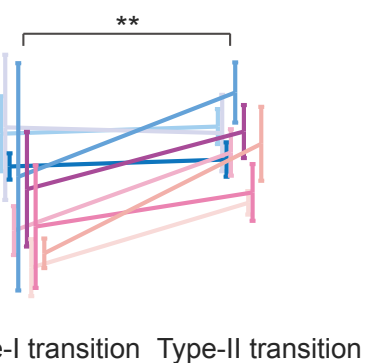

$\mathbf{F}$

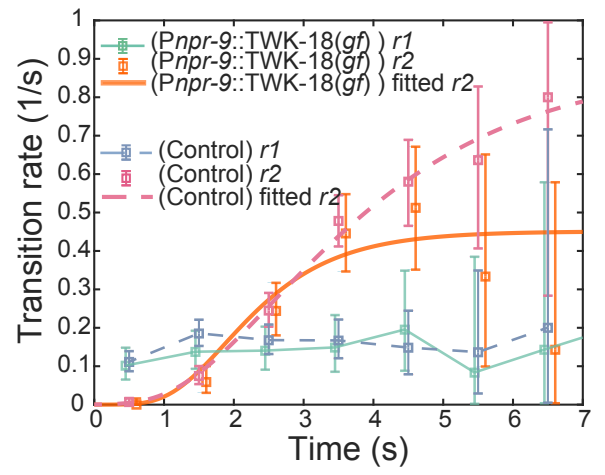


A

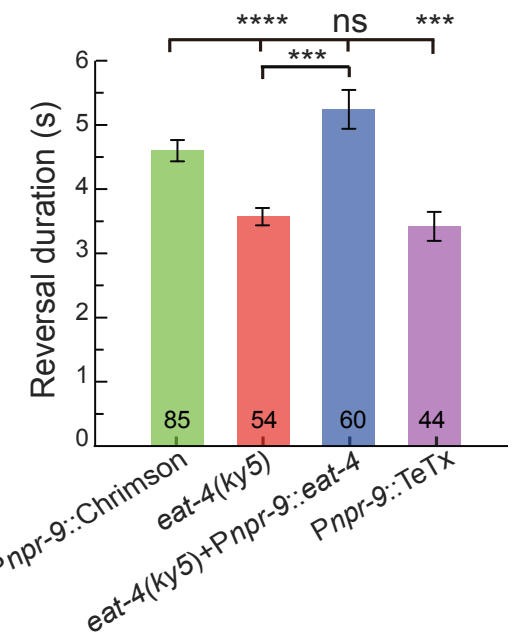

B

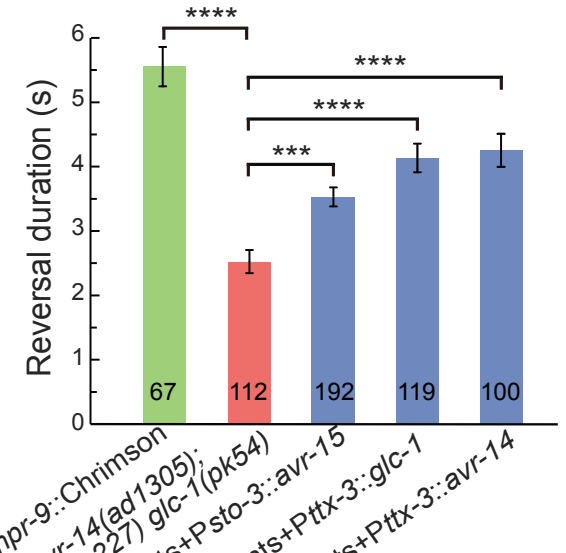

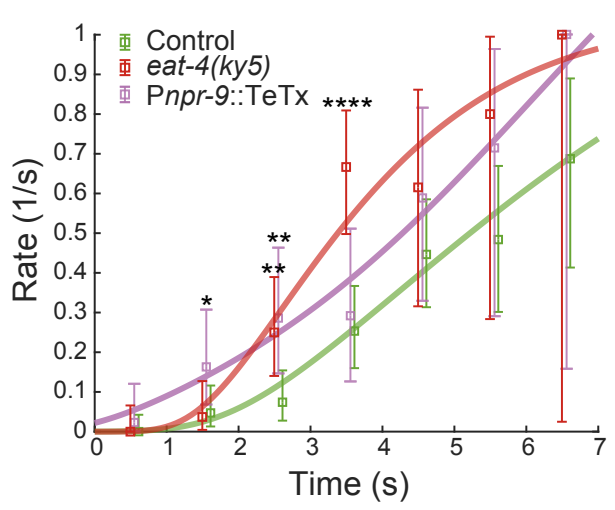

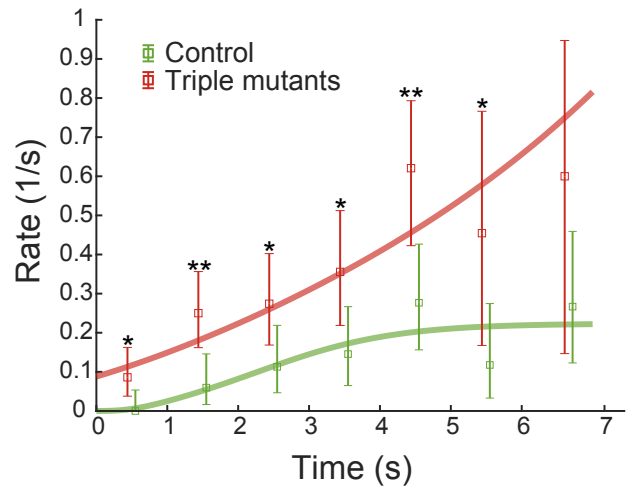

C

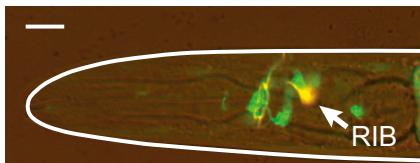

Pavr-15::GFP; Psto-3::mCherry

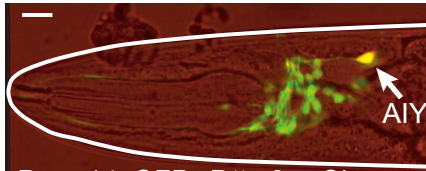

Pavr-14::GFP; Pttx-3::wCherry
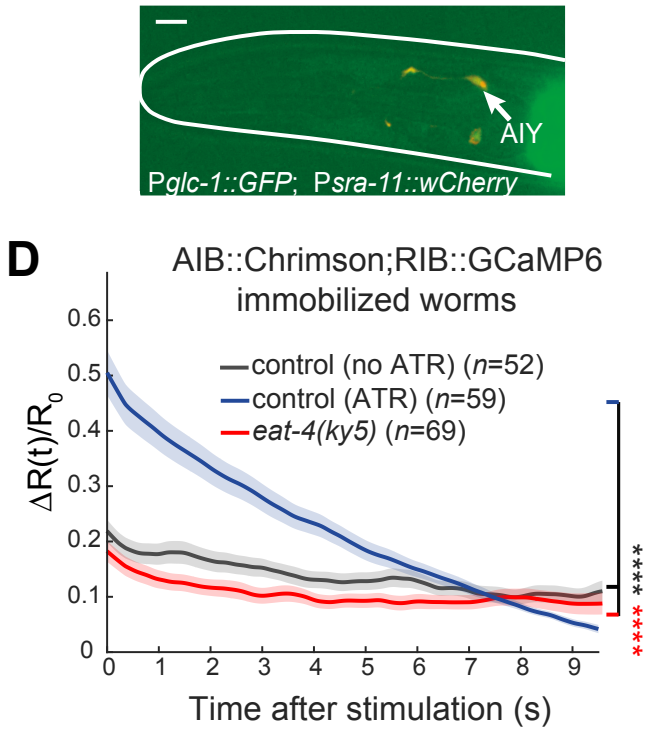

AIB activated; RIB Cell body
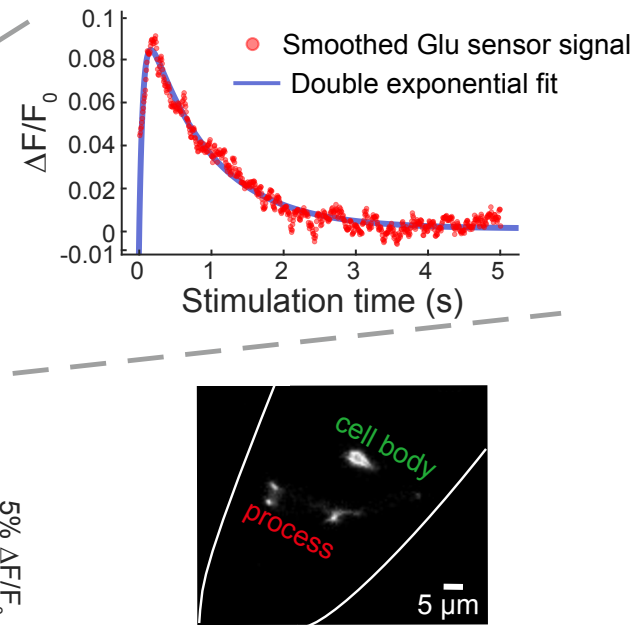

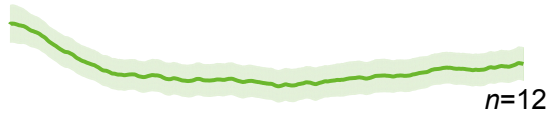

No ATR; RIB Process

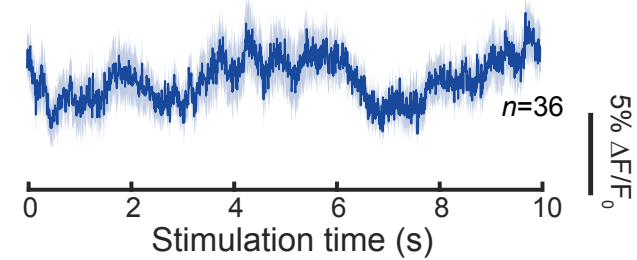


A

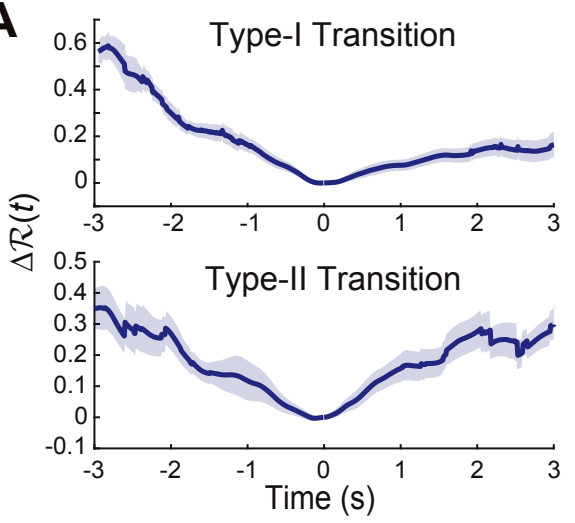

C

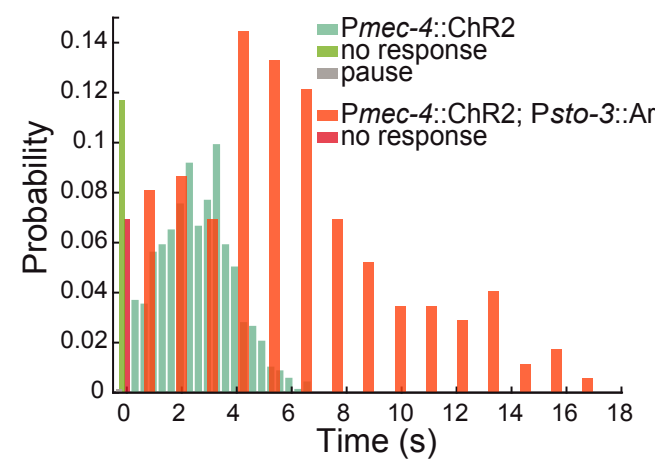

RIB::Arch

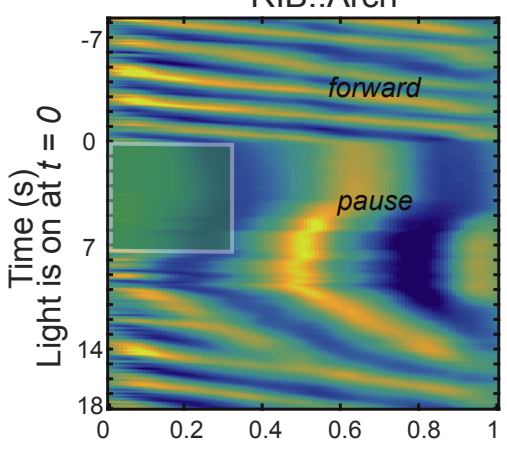

Fractional distance along the centerline (head $=0$; tail $=1$ )

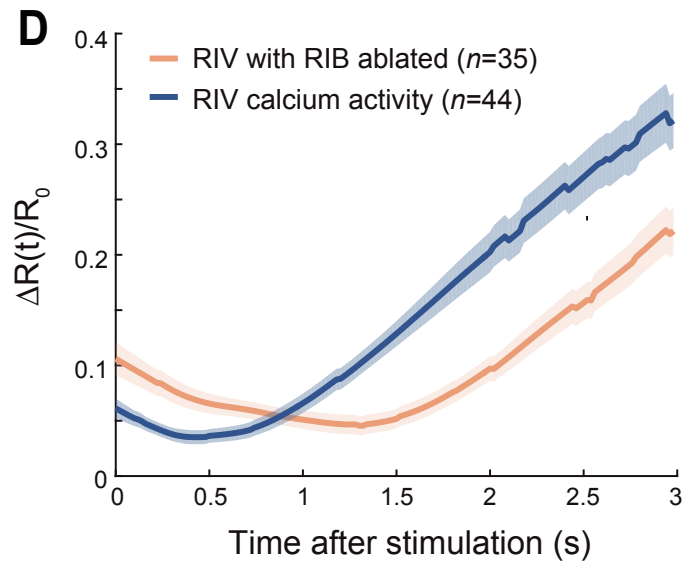

E

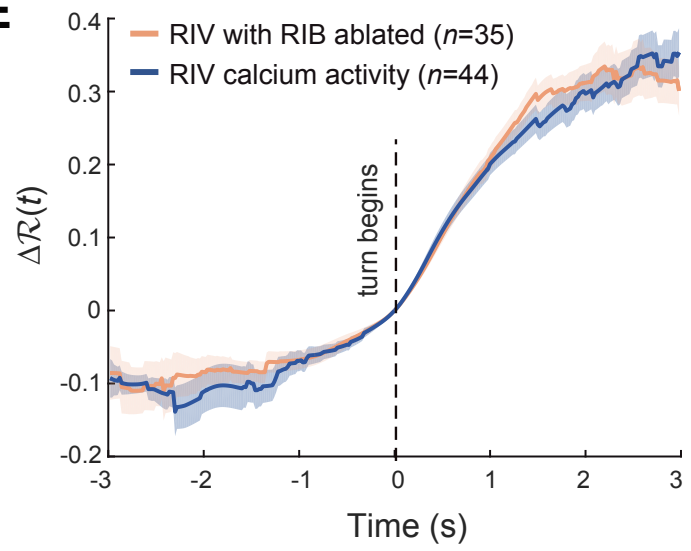

$(k \cdot L) \begin{array}{lllllllllll}-10 & -8 & -6 & -4 & -2 & 0 & 2 & 4 & 6 & 8 & 10\end{array}$

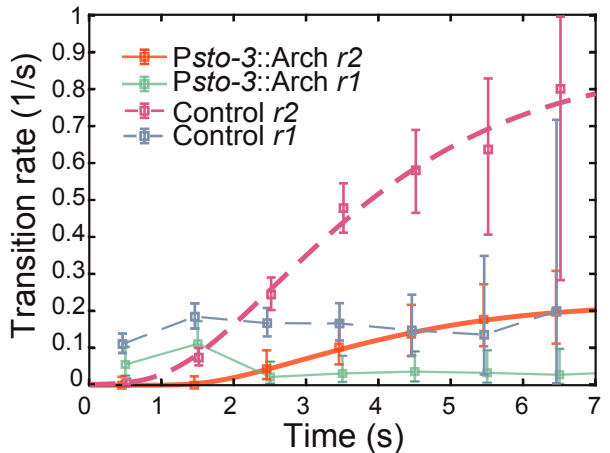

RIB::Chrimson ${ }^{1}$
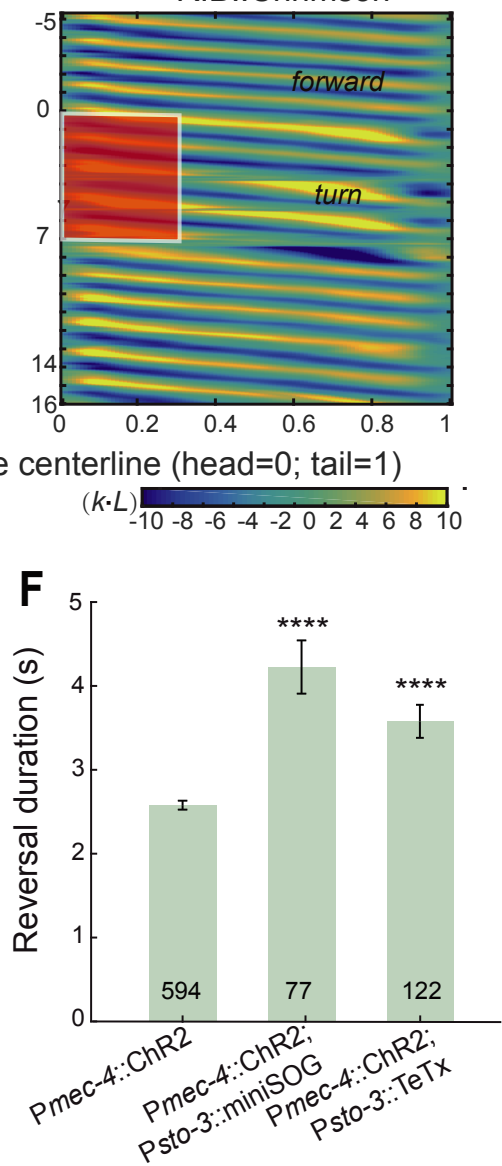
B

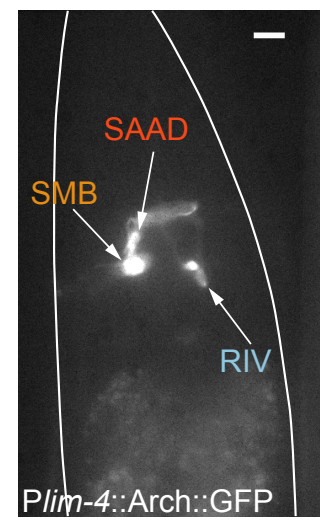

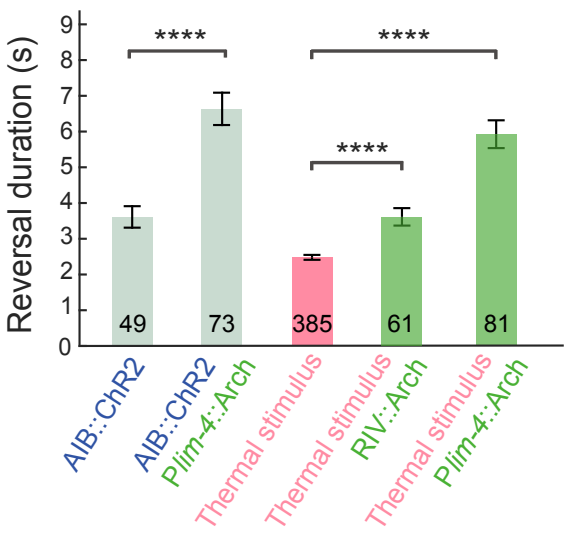

$\underset{79.6 \%}{\oplus} \underset{65.8 \%}{\bigoplus} \underset{49.9 \%}{\bigoplus} \underset{23.0 \%}{\bigoplus} \underset{20.9 \%}{\bigoplus}$ omega turn $\square$ omega turn $\square$ no turn
$0.75 \mathrm{~s}$ thermal stimulus

12s green laser

Thermal stimulus area

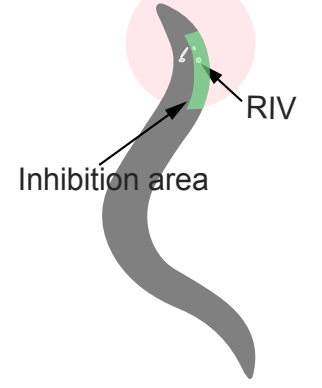

C

Pmec-4::ChR2; Plim-4::miniSOG ( $n=240)$
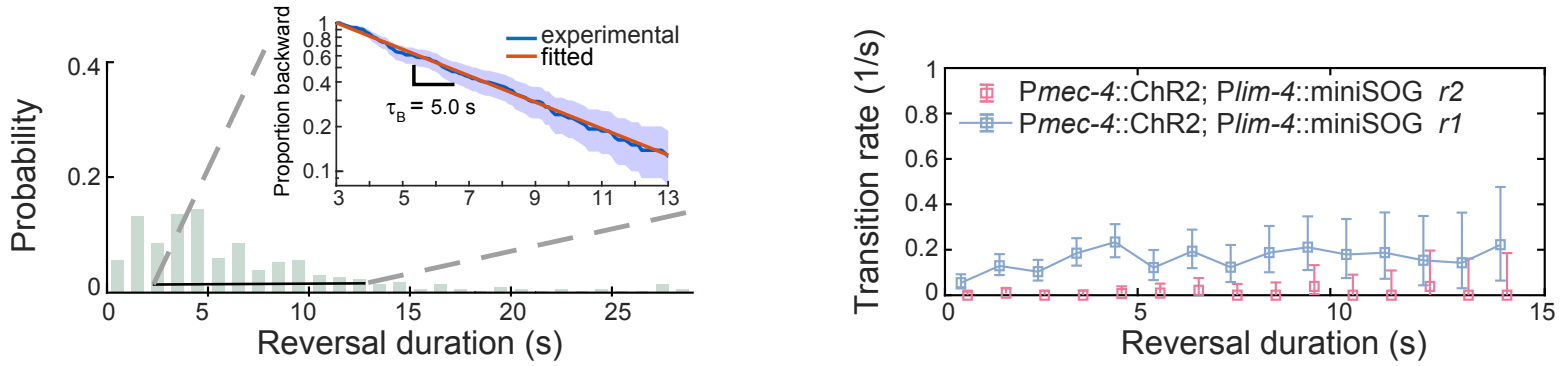

Wild-type $(n=1246)$
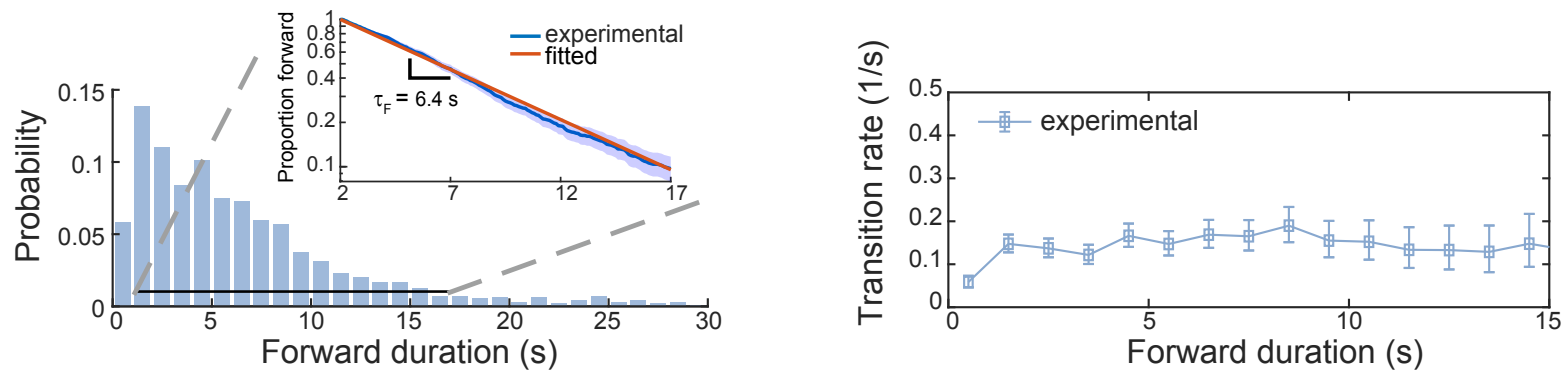


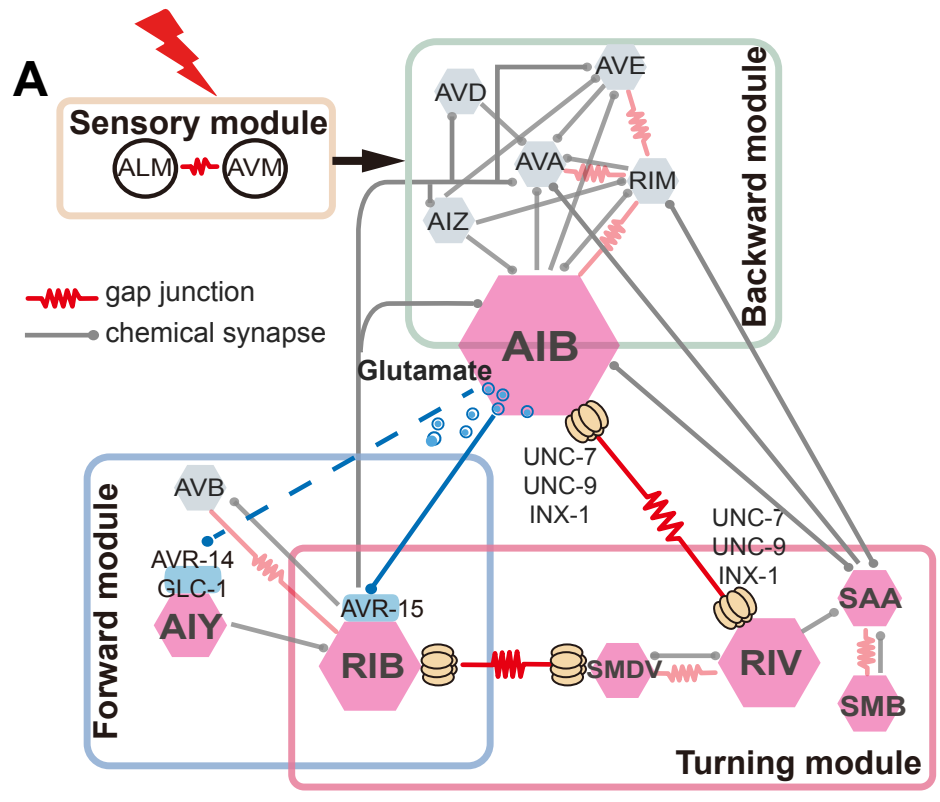

D

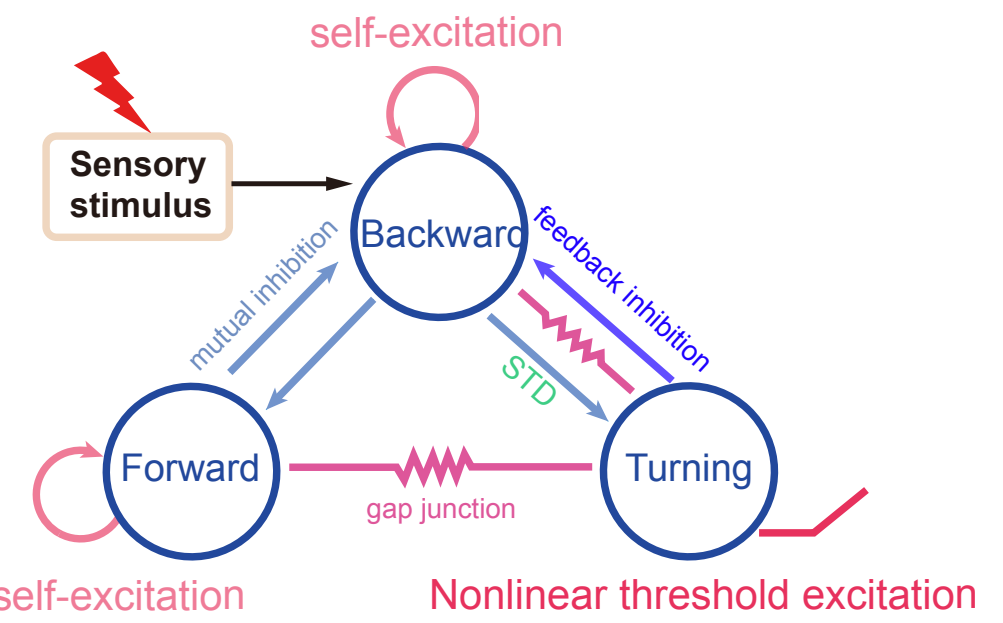

B

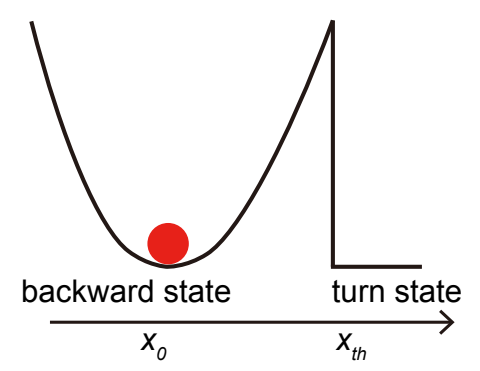

C

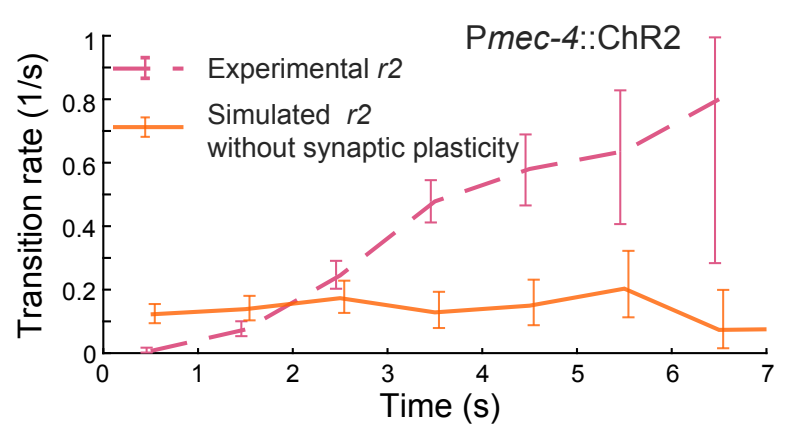

E

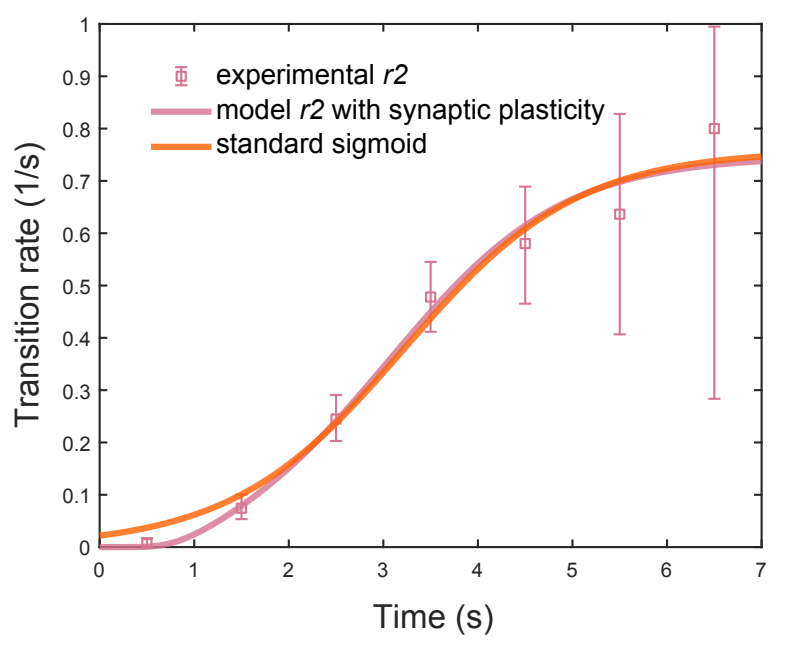


A

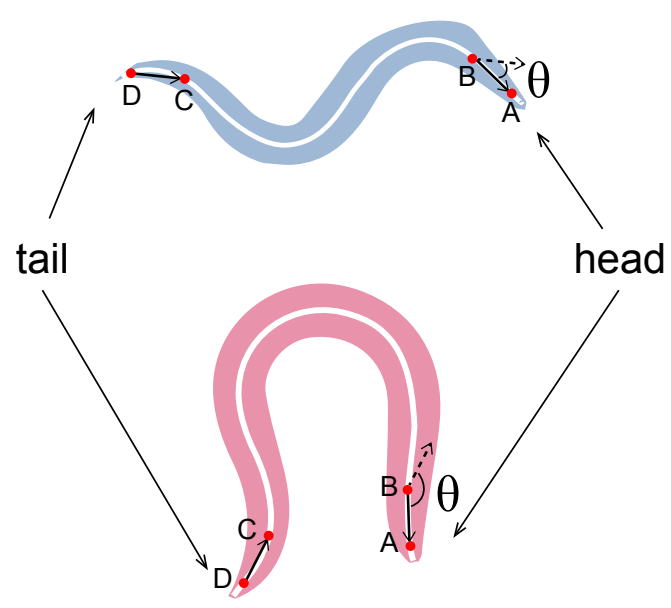

B

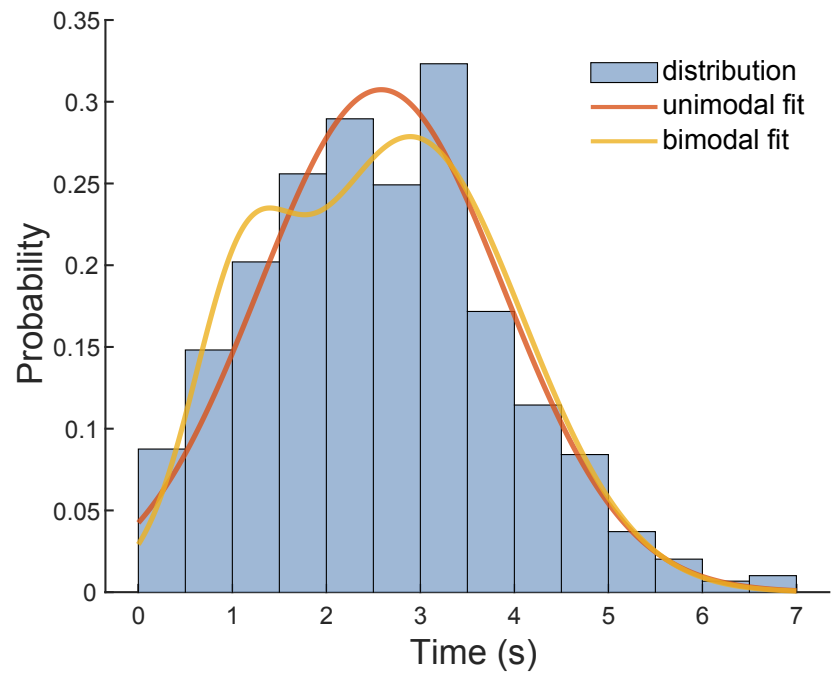

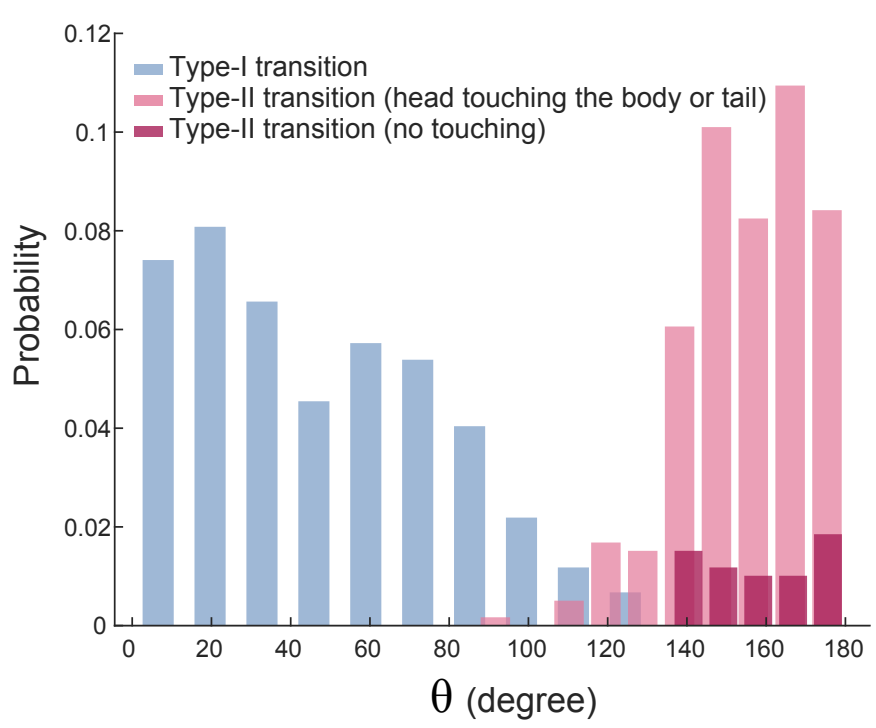

\begin{tabular}{|c|c|c|}
\hline Model & Unimodal & Bimodal \\
\hline Negative log likelihood & 997.23 & 986.02 \\
\hline P-value & \multicolumn{2}{|c|}{$5.3435 \mathrm{e}-05$} \\
\hline
\end{tabular}




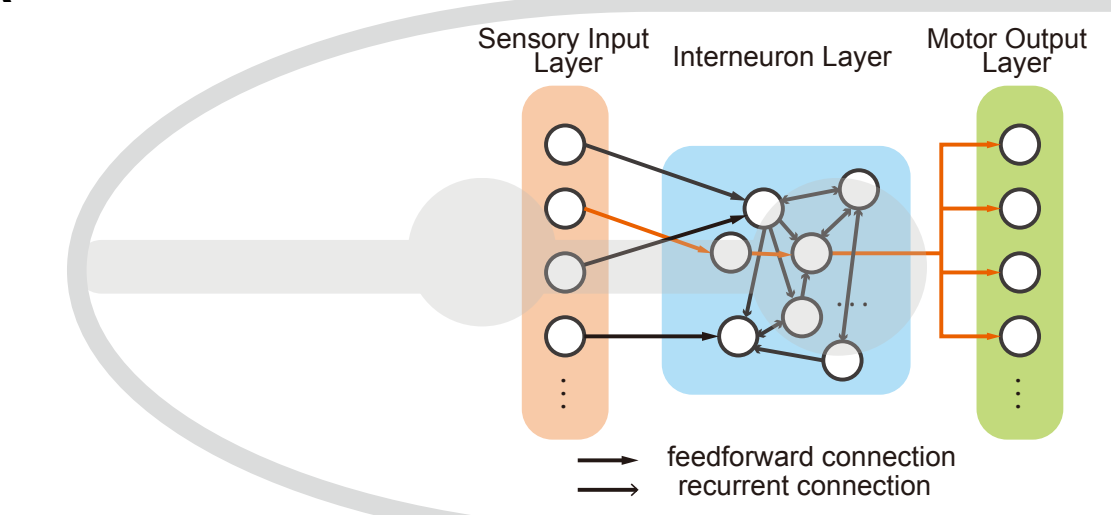

B
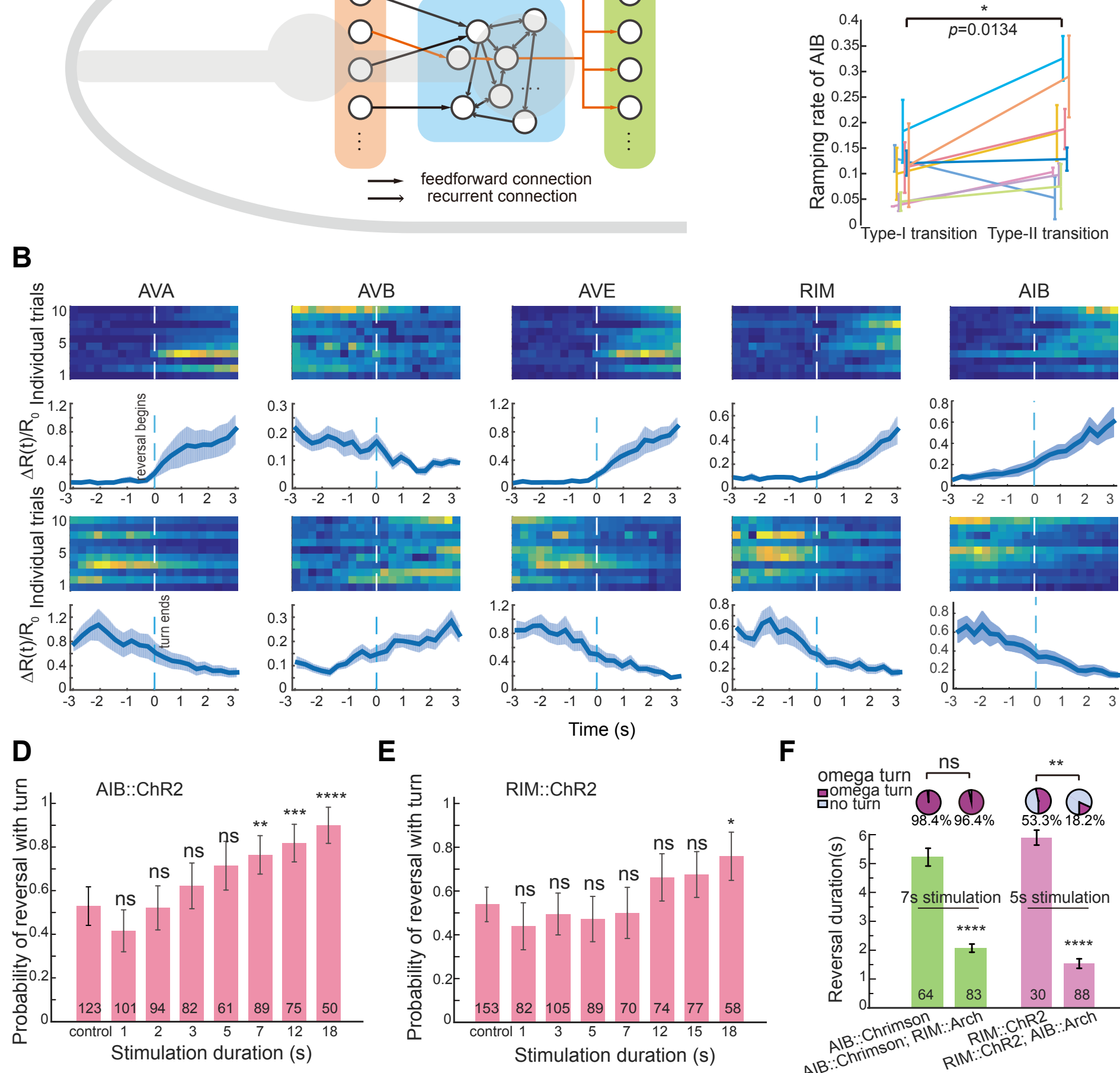

E
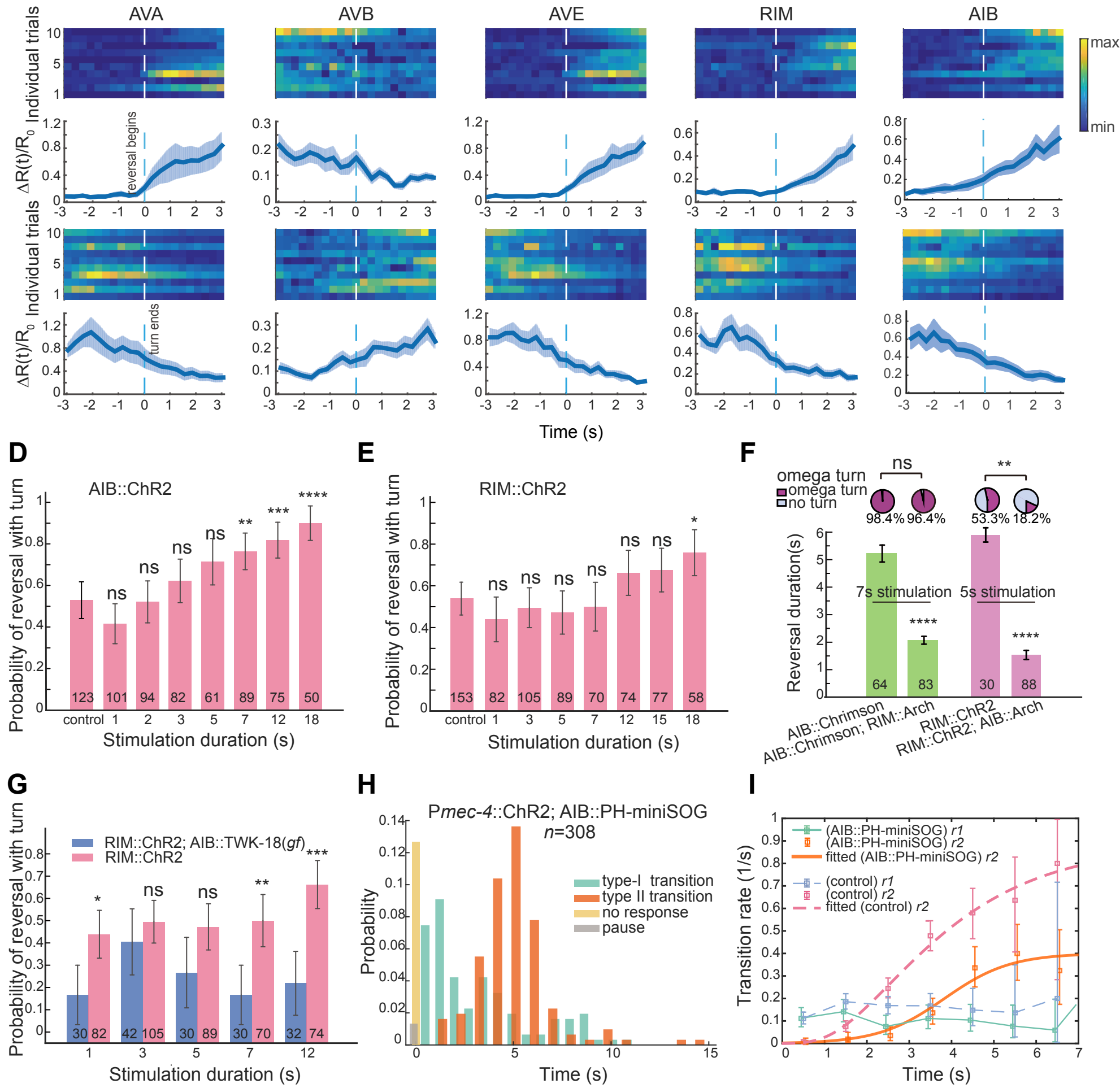
A RIV::GCaMP6 (spontaneous moving)

Type-I Transition

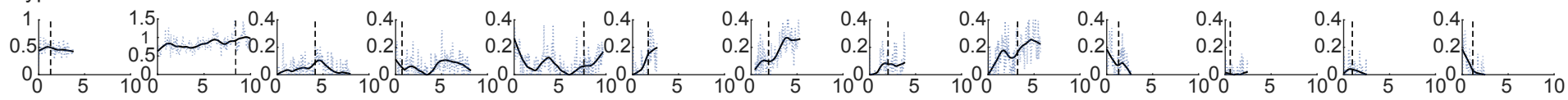

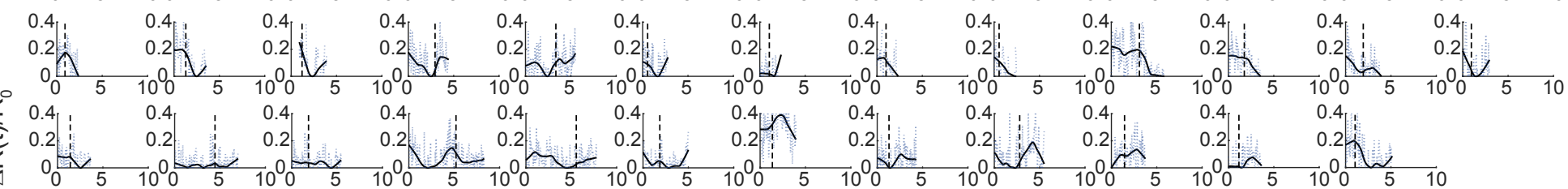

Type-II Transition

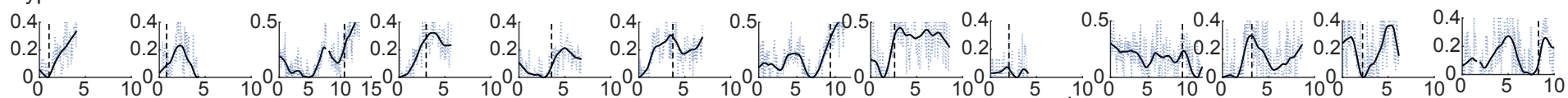
0.2
0

B

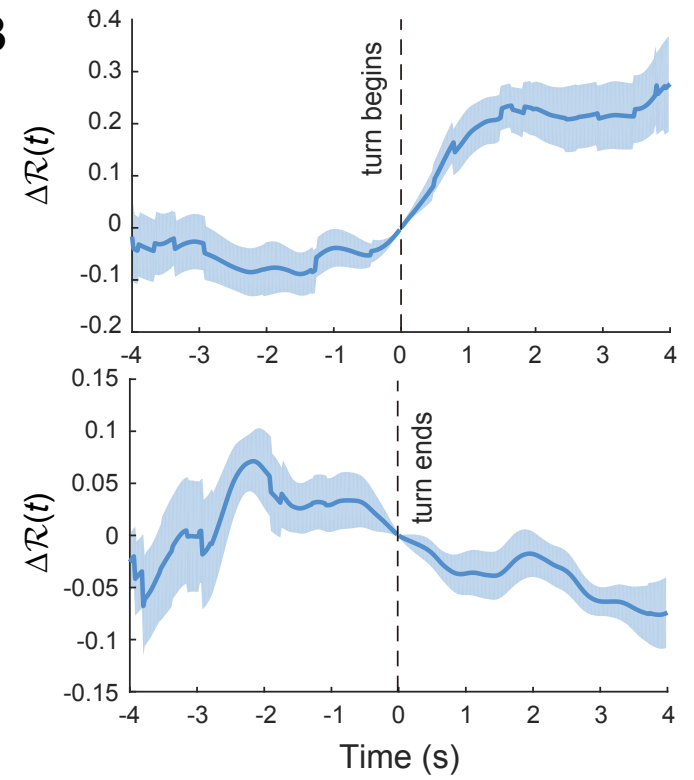

Time (s)

C AIB::Chrimson; RIV::GCaMP6 (immobilized)

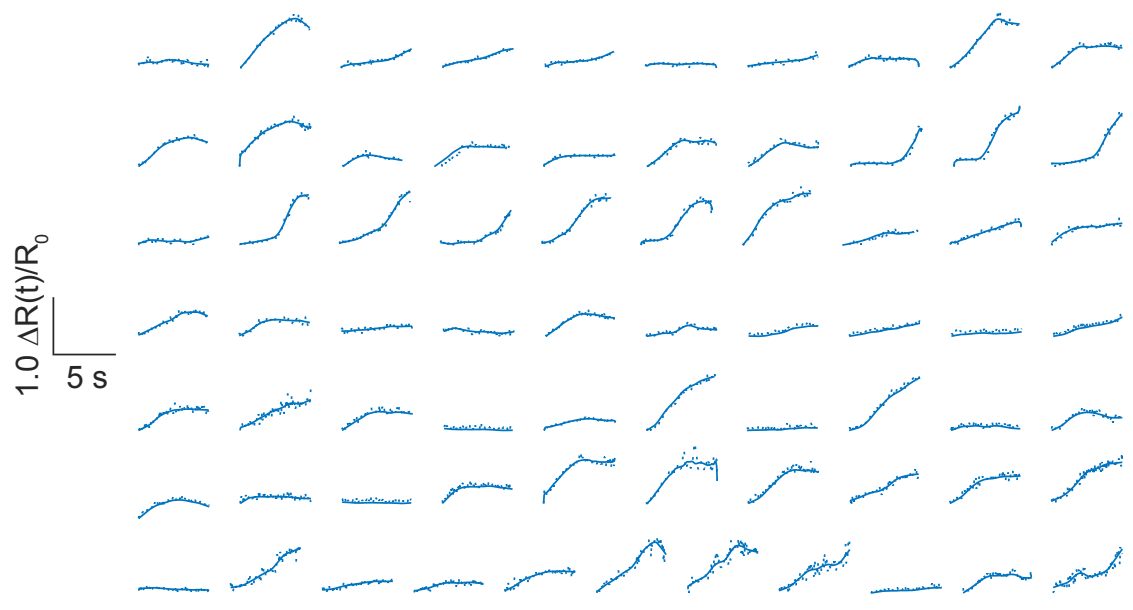

Time (s)

D inx-1 unc-7 unc-9; AIB::Chrimson; RIV::GCaMP6 (immobilized)

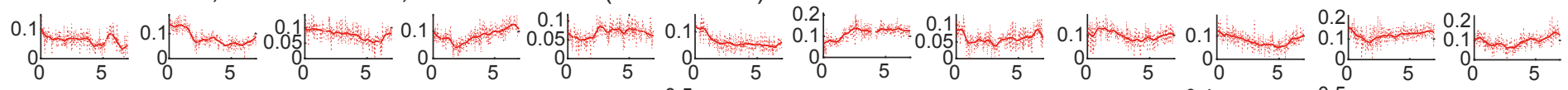

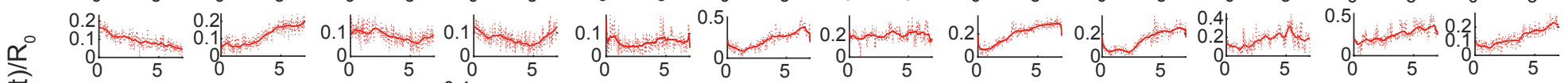

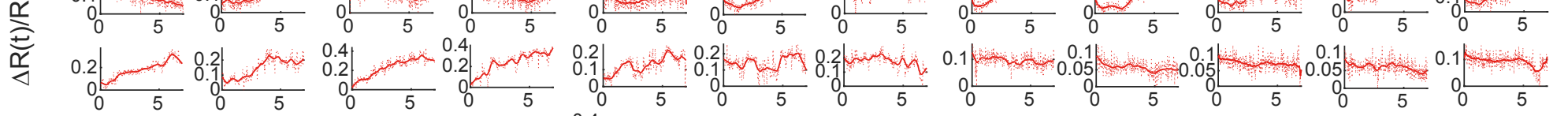
0.1
0.05

Time (s)

E AIB::Chrimson; RIV::Arch; RIV::GCaMP6 (immobilized)

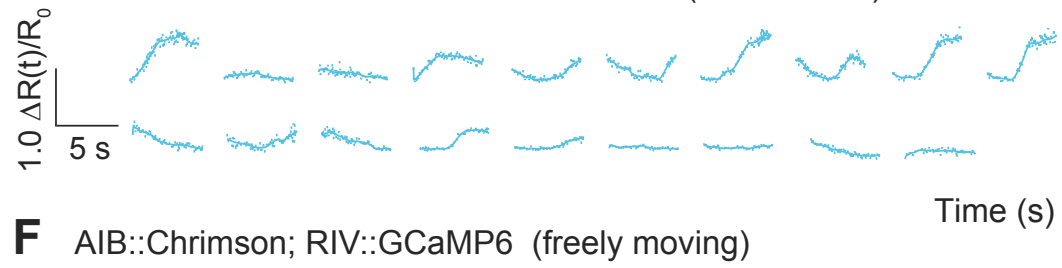

年

兴

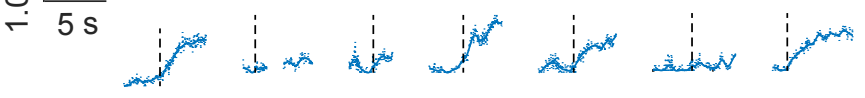

Time (s) 


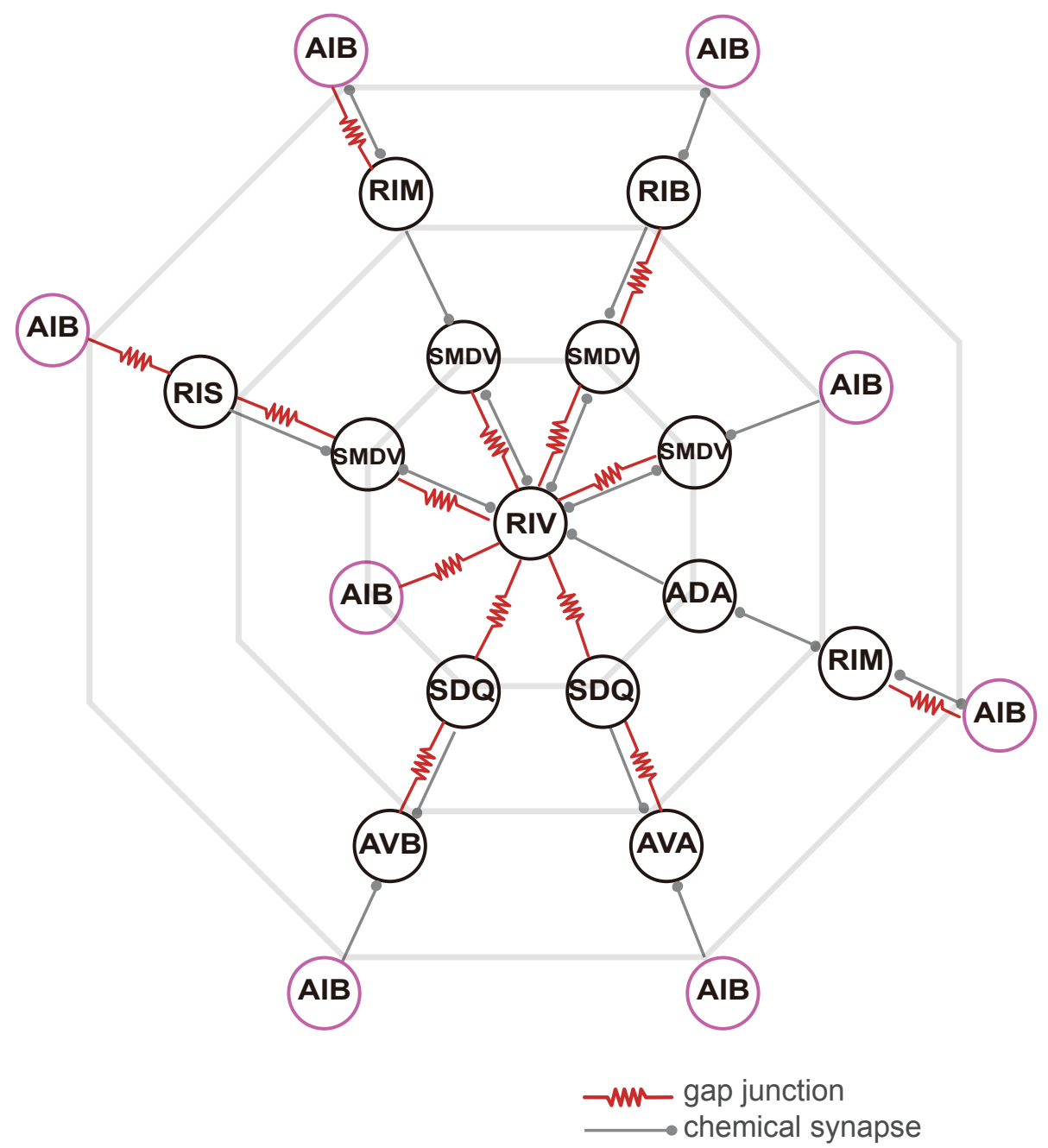




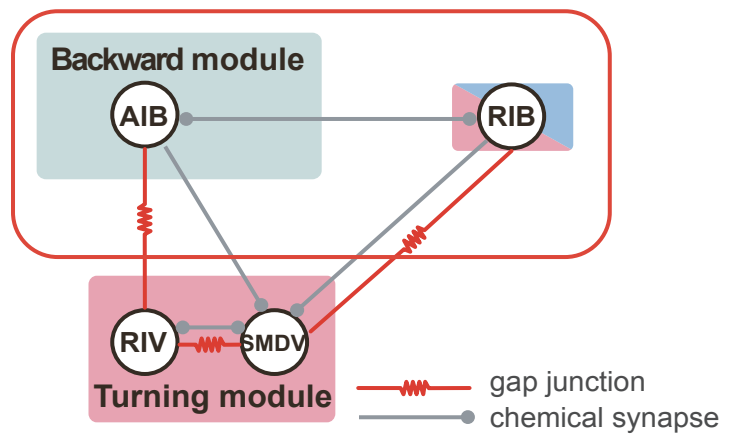

C AIB::Chrimson; RIB::GCaMP6 (control (ATR))

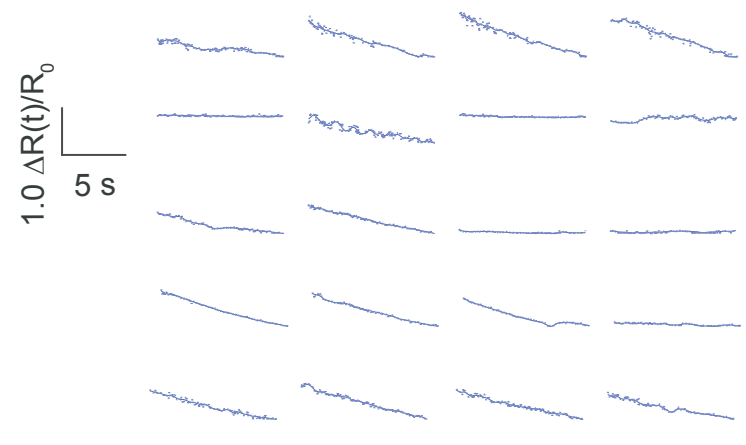

AIB::Chrimson; RIB::GCaMP6 (control (no ATR))

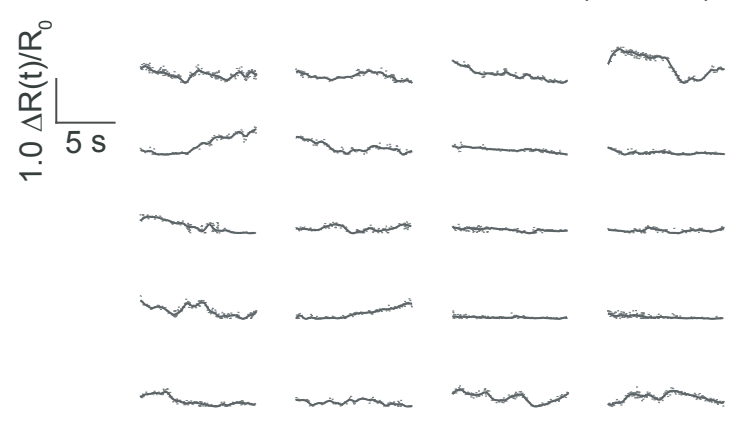

eat-4(ky5)III; AIB::Chrimson; RIB::GCaMP6
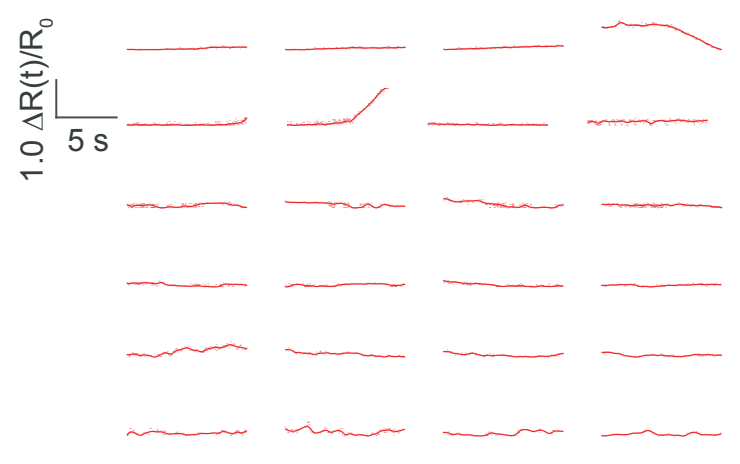

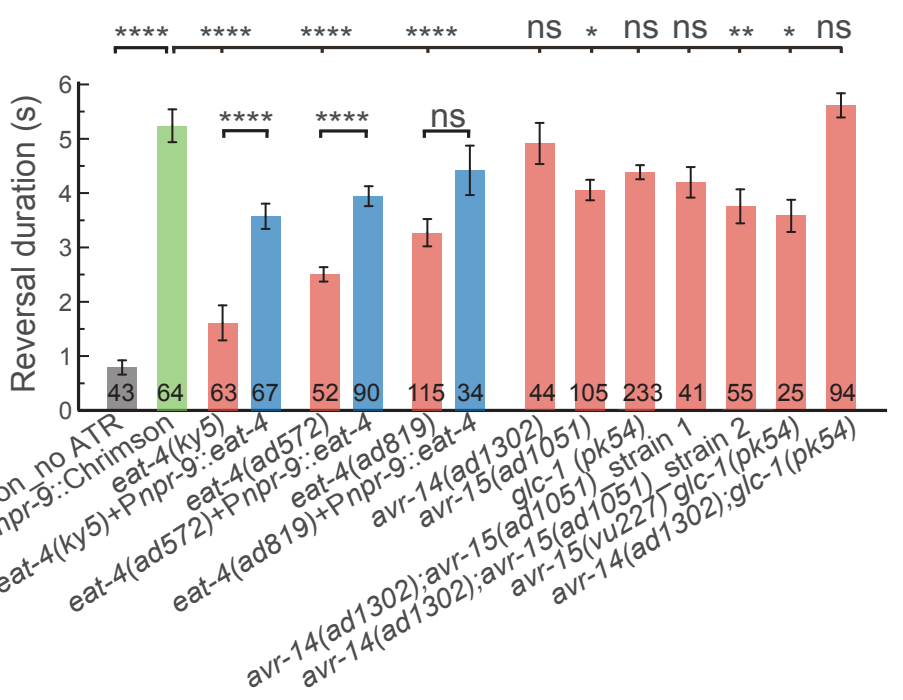


A AIB::Chrimson; RIB::GluSnFR, $n=25$
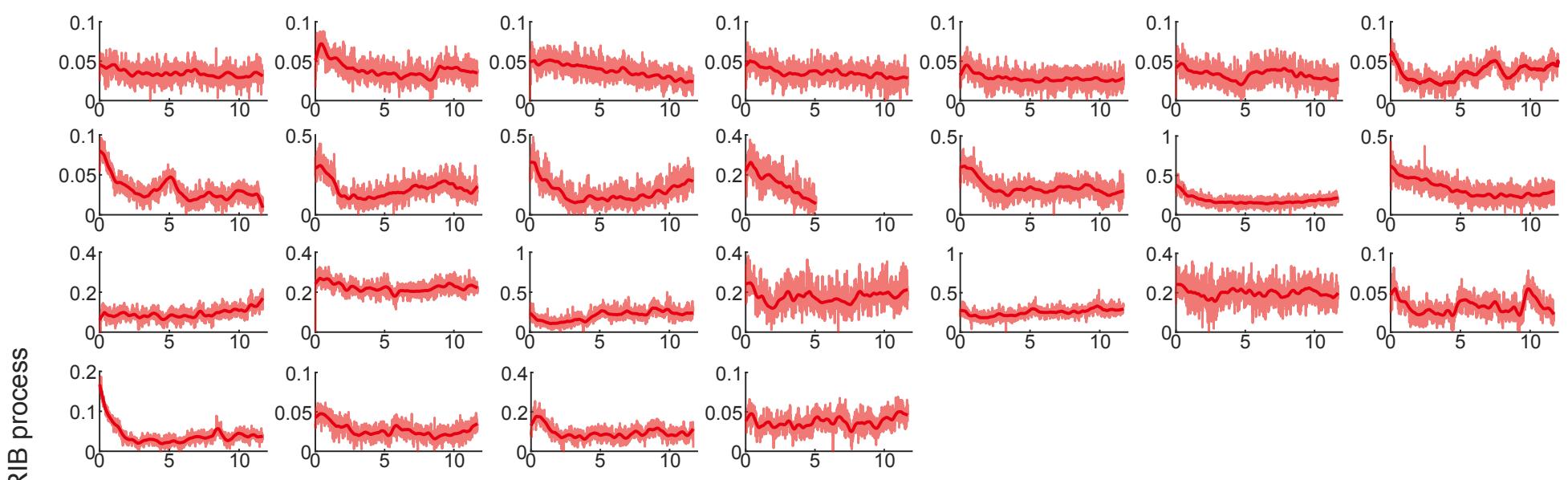

¿ eat-4(ky5)III; AIB::Chrimson; RIB::GluSnFR, $n=18$

$\stackrel{4}{4}$
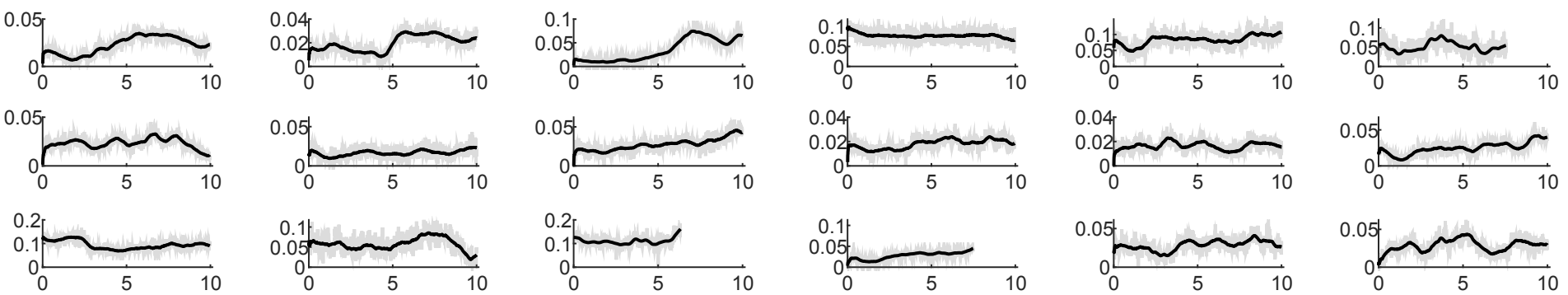

Time (s)

B AlB::Chrimson; AIB::GCaMP6, $n=21$

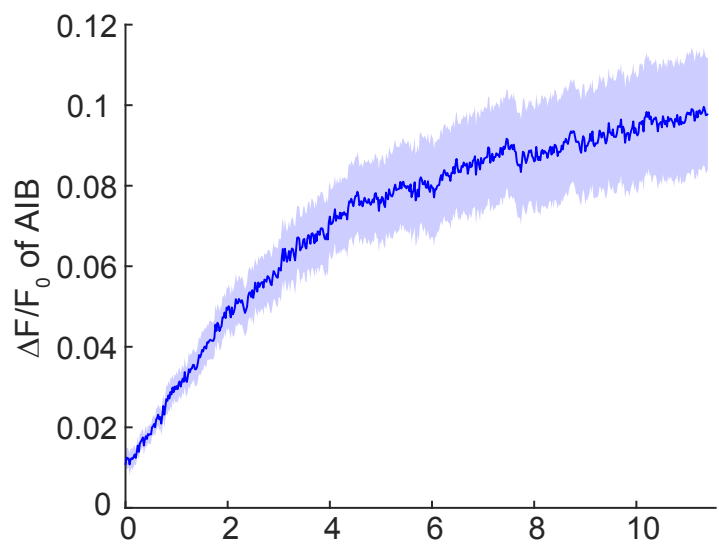

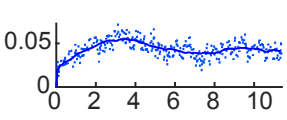
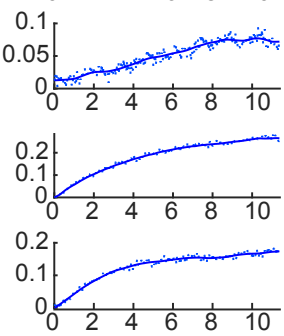

0.1

0.05 0.05

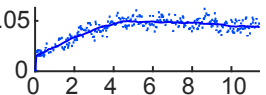

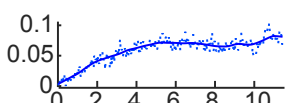

0.05
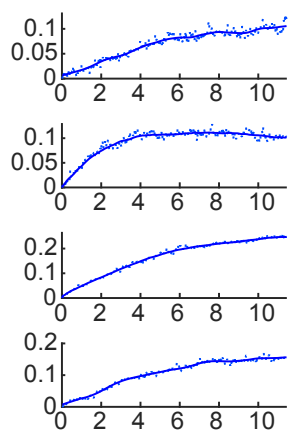

0.1
0.05
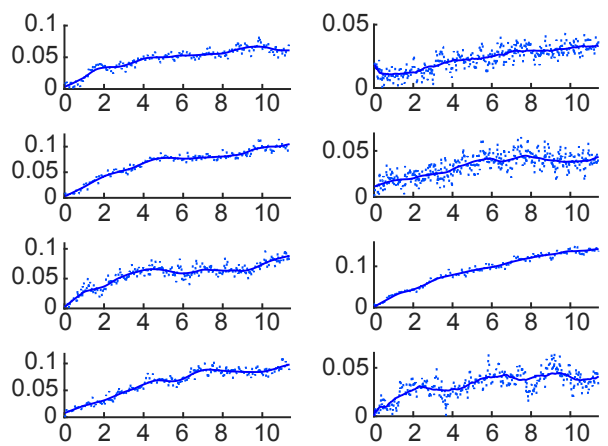

0.1

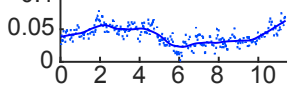

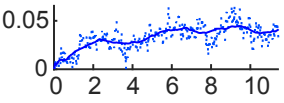

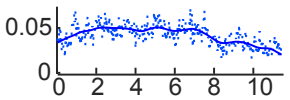

Time (s) 
A RIB::GCaMP6

Type-I Transition

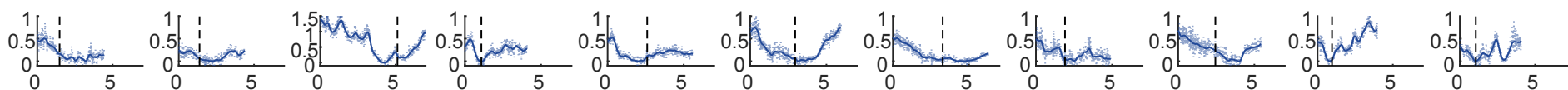

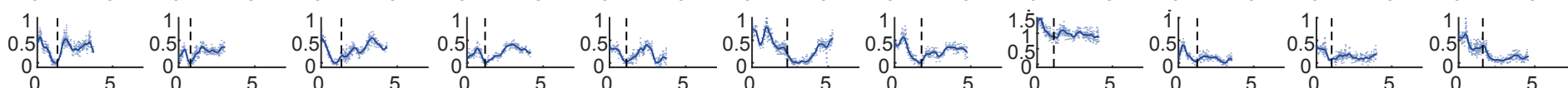

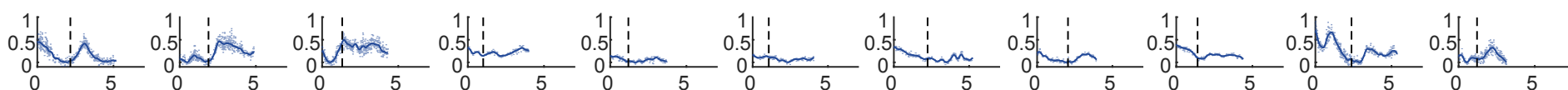

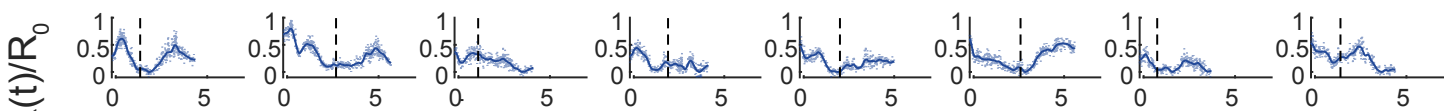

Type-II Transition

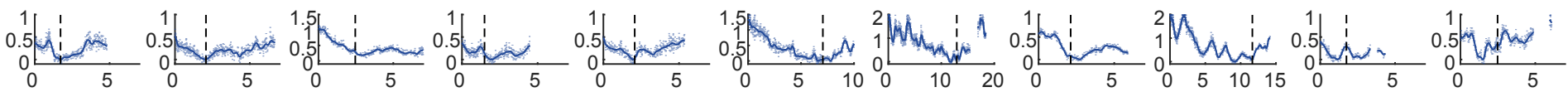

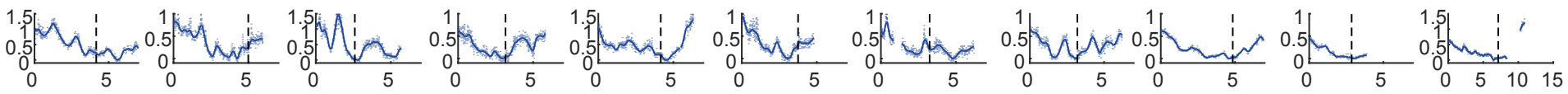

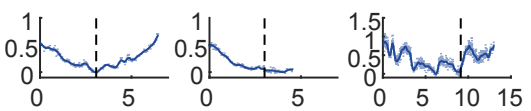

Time (s)
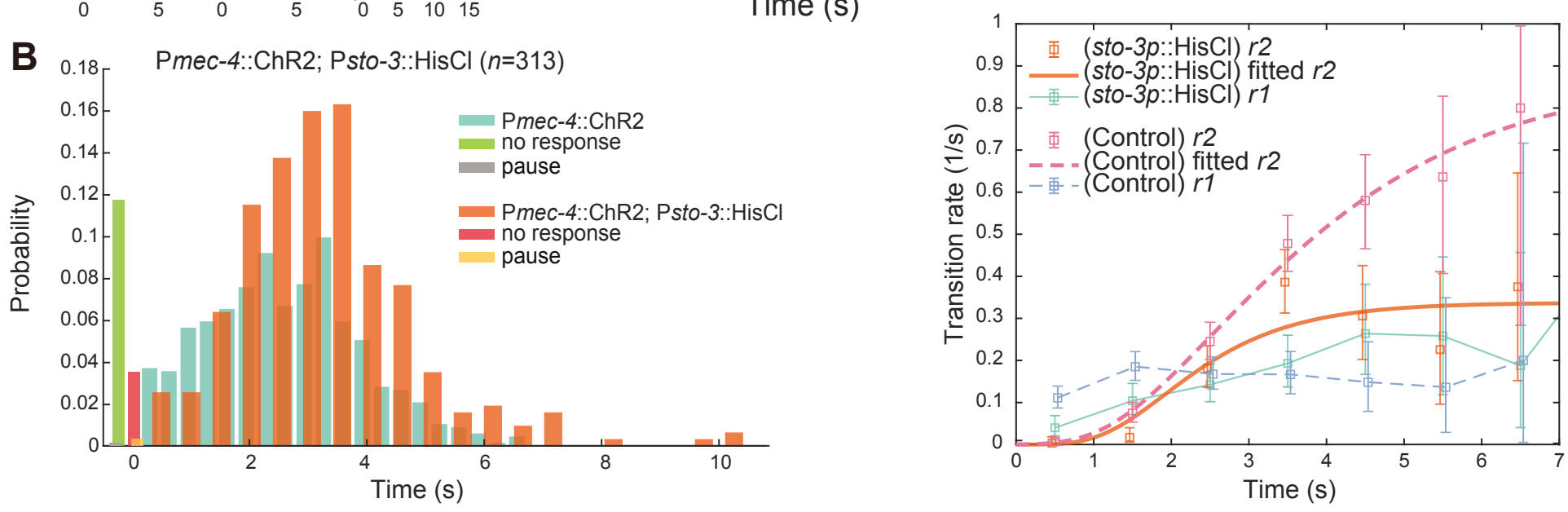

C AIB::Chrimson; Plim-4::GCaMP6; RIB::miniSOG (freely moving)

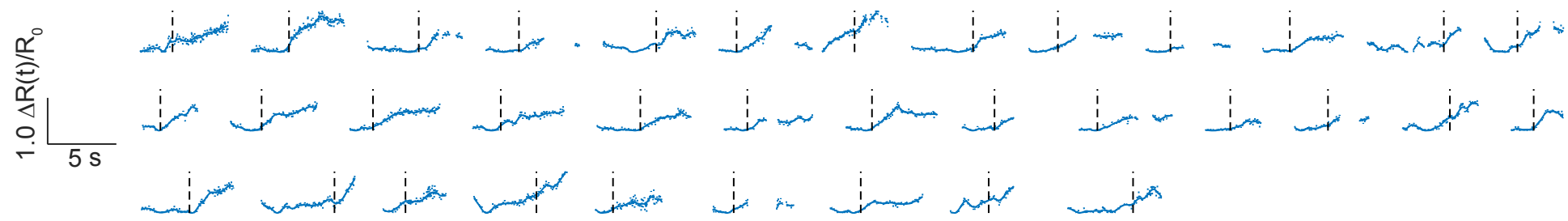

D AlB::Chrimson; Plim-4::GCaMP6 (freely moving)

Time (s)

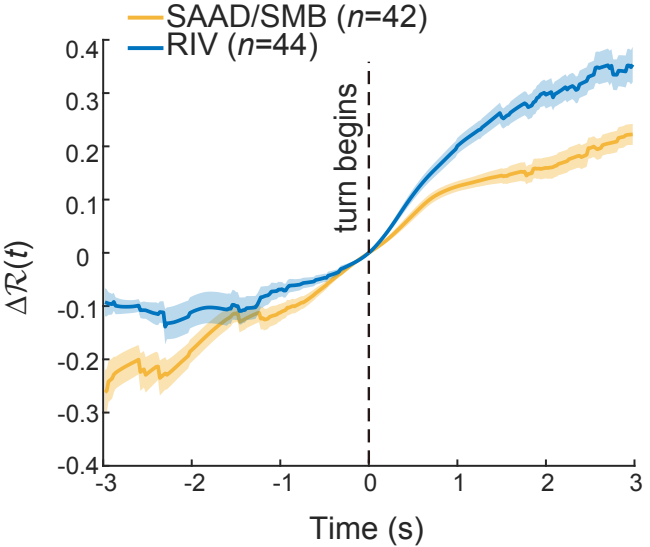

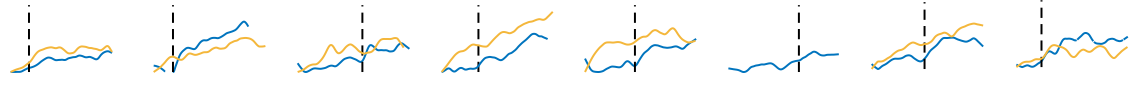

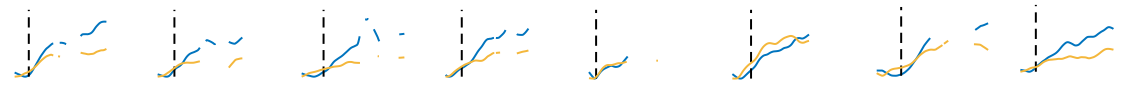

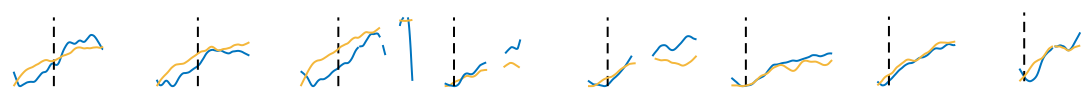

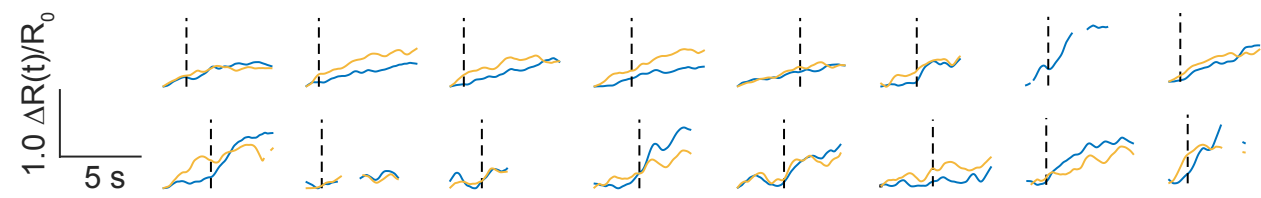
is

Time (s) 